Claremont Colleges

Scholarship@ Claremont

All HMC Faculty Publications and Research

HMC Faculty Scholarship

9-27-2018

\title{
Boundary Homogenization and Capture Time Distributions of Semipermeable Membranes with Periodic Patterns of Reactive Sites
}

\author{
Andrew J. Bernoff \\ Harvey Mudd College \\ Daniel Schmidt \\ Harvey Mudd College \\ Alan E. Lindsay \\ University of Notre Dame
}

\section{Recommended Citation}

Bernoff, Andrew J.; Schmidt, Daniel; and Lindsay, Alan E., "Boundary Homogenization and Capture Time Distributions of Semipermeable Membranes with Periodic Patterns of Reactive Sites" (2018). All HMC Faculty Publications and Research. 1155.

https://scholarship.claremont.edu/hmc_fac_pub/1155 


\title{
BOUNDARY HOMOGENIZATION AND CAPTURE TIME DISTRIBUTIONS OF SEMIPERMEABLE MEMBRANES WITH PERIODIC PATTERNS OF REACTIVE SITES*
}

\author{
ANDREW J. BERNOFF ${ }^{\dagger}$, ALAN E. LINDSAY , AND DANIEL D. SCHMIDT $^{\dagger}$
}

\begin{abstract}
We consider the capture dynamics of a particle undergoing a random walk in a halfspace bounded by a plane with a periodic pattern of absorbing pores. In particular, we numerically measure and asymptotically characterize the distribution of capture times. Numerically we develop a kinetic Monte Carlo (KMC) method that exploits exact solutions to create an efficient particlebased simulation of the capture time that deals with the infinite half-space exactly and has a run time that is independent of how far from the pores one begins. Past researchers have proposed homogenizing the surface boundary conditions, replacing the reflecting (Neumann) and absorbing (Dirichlet) boundary conditions with a mixed (Robin) boundary condition. We extend previous work to asymptotically determine the leakage parameter for the mixed boundary condition for arbitrary periodic pore configurations in the dilute fraction limit. In this asymptotic limit, we pose and solve an optimization problem for the Bravais lattice which maximizes the capture rate of the absorbing pores, finding the hexagonal lattice to be the global maximum.
\end{abstract}

Key words. Brownian motion, Berg-Purcell, singular perturbations, boundary homogenization, Monte Carlo methods

AMS subject classifications. 35B25, 35C20, 35J05, 35J08

DOI. $10.1137 / 17 \mathrm{M} 1162512$

1. Introduction. We consider the problem of describing the arrival times of diffusing particles to a planar surface with a periodic array of absorbing pores and reflecting otherwise. This problem arises in a variety of important applications. For example, the design and usage of semipermeable membranes is crucial in industrial processes such as filtration [44], gas separation [3], and the design of fuel cells [47]. In cellular-scale biological processes, cells need to accurately determine ambient chemical concentrations through encounters with diffusing signaling molecules at receptors distributed on an otherwise inert membrane $[36,41,56]$. In each of the above examples, the common problem is to describe the rate of transport through, or the reaction rate on, a mostly impermeable surface with localized reactive patches.

The general problem takes the form of a diffusion equation where for $\mathbf{x}=(x, y, z) \in$ $\mathbb{R}^{3}$, the quantity $p(\mathbf{x}, t)$ is the probability that a particle originating at $\mathbf{x}_{0}$ is free at $\mathbf{x}$ at time $t$. This distribution solves

$$
\begin{gathered}
\frac{\partial p}{\partial t}=D \Delta p, \quad \mathbf{x} \in \Omega, \quad t>0 ; \quad p(\mathbf{x}, 0)=\delta\left(\mathbf{x}-\mathbf{x}_{0}\right), \quad \mathbf{x} \in \Omega ; \\
p=0 \quad \text { on } \quad \mathbf{x} \in \Gamma_{a} ; \quad D \nabla p \cdot \hat{\mathbf{n}}=0 \quad \text { on } \quad \mathbf{x} \in \Gamma_{r},
\end{gathered}
$$

where $D$ is the diffusivity of the particle, and the domain $\Omega$ is a subset of $\mathbb{R}^{3}$ whose boundary, $\partial \Omega$, is partitioned into an absorbing set of pores, $\Gamma_{a}$, whose impermeable

${ }^{*}$ Received by the editors December 26, 2017; accepted for publication (in revised form) July 27, 2018; published electronically September 27, 2018.

http://www.siam.org/journals/mms/16-3/M116251.html

Funding: The second author's research was supported by NSF grants DMS-1516753 and DMS1815216. The first author's research was supported by Simons Foundation grant 317319.

${ }^{\dagger}$ Department of Mathematics, Harvey Mudd College, Claremont, CA 91711 (ajb@hmc.edu, danielschmidt24@gmail.com).

${ }^{\ddagger}$ Department of Applied and Computational Math \& Statistics, University of Notre Dame, South Bend, IN 46656 (a.lindsay@nd.edu). 


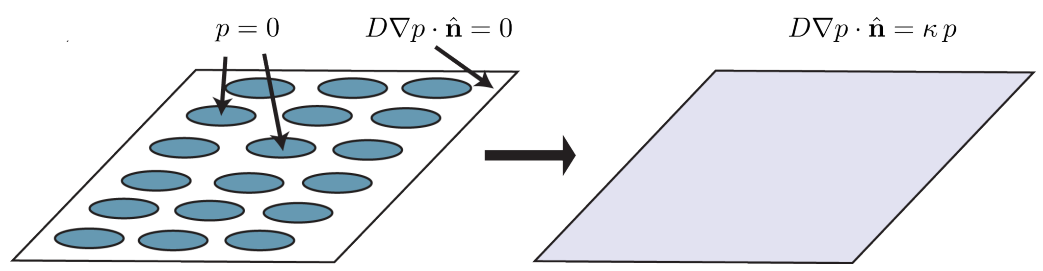

FIG. 1. We seek to replace the heterogeneous boundary conditions (1.1b) with the homogeneous, semiabsorbing boundary condition (1.3).

complement, $\Gamma_{r}$, is reflecting. We choose $\hat{\mathbf{n}}$, the normal to the surface $\partial \Omega$, to point into the bulk.

For this paper, we will consider $\Omega$ to be the half-space $z>0$ whose boundary $\partial \Omega$ (at $z=0$ ) is partitioned into a doubly periodic array of absorbing pores, $\Gamma_{a}$, whose complement, $\Gamma_{r}$, is reflecting. A longstanding problem is to describe the role that the pore size distribution and spatial organization of the absorbing set $\Gamma_{a}$ has on the capture rate. A history of important results towards this goal is in section 2 .

This problem offers several challenges. First, the unbounded domain on which we consider the system (1.1) leads to long capture times; to see this, suppose that the entire plane is absorbing (i.e., $\Gamma_{a}=\partial \Omega$ ). In this case, the distribution of capture times, $\rho(t)$, for a particle released at a height $z_{0}$ can be computed explicitly (cf. (3.3)):

$$
\rho(t)=\frac{z_{0}}{2 \sqrt{\pi D} t^{3 / 2}} e^{-\frac{z_{0}^{2}}{4 D t}},
$$

which decays as $t^{-3 / 2}$ at large times. While all the particles will eventually be captured, the fat tail of the distribution means that the mean capture time is infinite. This precludes the use of many analytical techniques (such as eigenvalue decompositions) and numerical methods (such as standard Monte Carlo techniques).

This problem is also challenging due to the mixture of Neumann and Dirichlet boundary conditions (1.1b) on the membrane which precludes many of the classical methods for linear PDEs. The method of boundary homogenization, or effective medium theory $[7,16,25,32]$, is an alternate approach which seeks to replace the mixed boundary conditions (1.1b) with a single uniform boundary condition (see Figure 1):

$$
D \nabla p \cdot \hat{\mathbf{n}}=\kappa p \quad \text { on } \quad \partial \Omega .
$$

Heuristically this works because an ensemble of particles that originate at a point far above the surface will spread over a large swath of the pore geometry. The capture probability at that point (i.e., solutions of (1.1)) will only reflect the average properties on that swath. In other words, the capture time of a particle far from the surface depends only on some homogenized properties of the particular spatial configuration of the surface absorbers. The main challenge is therefore to determine the leakage or permeability parameter, $\kappa$, that best represents the homogenized capture properties of the periodic pore configuration. We review past results for the homogenized theory in section 2 .

In the present work, we consider periodic arrays of absorbing pores. We begin by considering two-dimensional Bravais lattices. From two linearly independent primitive vectors $\mathbf{p}, \mathbf{q}$ in $\mathbb{R}^{2}$, the Bravais lattice is defined as

$$
\Lambda=\left\{m \mathbf{p}+n \mathbf{q} \mid(m, n) \in \mathbb{Z}^{2}\right\} .
$$




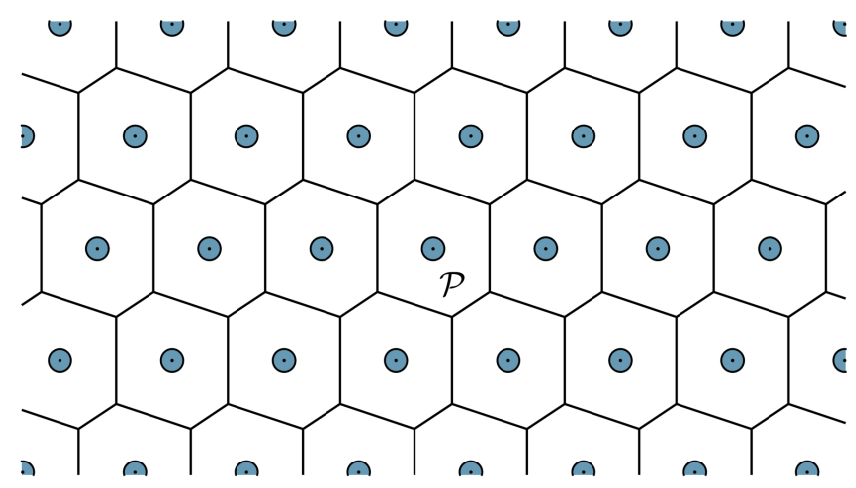

FIG. 2. A Bravais lattice (1.4) of circular pores with $\mathbf{p}=\left(\frac{1}{3}, 1\right), \mathbf{q}=(1,0)$ and Wigner-Seitz cell $\mathcal{P}$. This is an example of a homogeneous lattice which has uniform pore sizes.

Associated to each point in the Bravais lattice is a primitive cell, $\mathcal{P}$, containing a point in the lattice $\Lambda$; one way to define $\mathcal{P}$ is to construct the Voronoi cell associated to each element of $\Lambda$ defined as the set of points closer to it than any other element of $\Lambda$. This also leads to a natural definition of the boundary of the cell, $\partial \mathcal{P}$, as the set of points for which two (or more) elements of $\Lambda$ are closest. This construction leads to a tiling of the plane by a set of congruent polygons which are known in crystallography as the Wigner-Seitz cells. The area of the primitive cell is $|\mathcal{P}|=|\mathbf{p} \times \mathbf{q}|$.

In Figure 2, we show an example of a Bravais lattice and the associated WignerSeitz cells. The simplest pore configuration has a single circular pore in the primitive cell $\mathcal{P}$ centered at each point of the Bravais lattice; we refer to this as a homogeneous lattice configuration. More generally, one can partition the primitive cell into attracting and repelling sets. We will consider examples of two such geometries: stripes and a cell containing multiple circular pores with different radii, which we will refer to as heterogeneous lattice configurations.

It is convenient to nondimensionalize the diffusion problem (1.1) for the specific geometry considered here. We introduce a lengthscale that is the square root of the area of the primitive cell, $L=\sqrt{|\mathcal{P}|}$, and a timescale $T=L^{2} / D$. Rescaling yields

$$
\begin{gathered}
\frac{\partial p}{\partial t}=\Delta p, \quad z>0, \quad t>0 ; \quad p(\mathbf{x}, 0)=\delta\left(\mathbf{x}-\mathbf{x}_{0}\right), \quad z>0 \\
p=0 \quad \text { on } \quad \mathbf{x} \in \Gamma_{a} ; \quad p_{z}=0 \quad \text { on } \quad \mathbf{x} \in \Gamma_{r},
\end{gathered}
$$

where $\Gamma_{a}$ and $\Gamma_{r}$ are a partition of the plane $z=0$ into absorbing and reflecting sets with the symmetries of a Bravais lattice. Moreover, the area of the primitive cell of the lattice is unity, $|\mathcal{P}|=1 .^{1}$ The nondimensional version of the homogenized boundary condition can now be written as

$$
p_{z}=\bar{\kappa} p \quad \text { on } \quad z=0 \text {, }
$$

where we define the nondimensional leakage parameter $\bar{\kappa}=\frac{\kappa L}{D}$.

Within this setting, the present work makes several contributions. First, in section 3, we develop and validate a novel kinetic Monte Carlo (KMC) method for the

\footnotetext{
${ }^{1}$ The reader is cautioned that at various points we retain $|\mathcal{P}|$ in formulae for the sake of insight and clarity, even though it has been nondimensionalized to unity.
} 
rapid solution of (1.5). This method is based on a two-stage algorithm which alternates between projecting the diffusing particle from the bulk to the surface and vice versa. At each stage, the spatial location and duration of the particle's excursion are drawn from exact distributions, which allows for large jumps and rapid sampling of the capture time distribution. Simulations of up to $10^{9}$ particles allow us to accurately estimate the leakage parameter $\bar{\kappa}$ in the boundary condition (1.6). Notably, this method bypasses many complications which arise from particle simulations on unbounded domains and the fat tails associated with distributions such as (1.2).

In section 4, we review homogenization as it applies to the periodic half-plane problem (1.5) and derive an exact solution for this problem with the homogenized boundary condition (1.6). We develop a strategy for determining the best fit for the leakage parameter, $\bar{\kappa}$, from our numerical simulations. Past studies have considered a finite height domain for which the mean capture time is finite and inversely proportional to $\bar{\kappa}[4,9,10,32]$. As our domain is unbounded, and the mean capture time is infinite, a more nuanced approach is needed. We determine $\bar{\kappa}$ by minimizing the mean absolute error over the entire capture time distribution. We validate these ideas by considering absorbing stripes for which an exact solution to the homogenized theory is known (an idea introduced and employed in [32]).

A second contribution, described in section 5, is a high-order asymptotic estimate for $\bar{\kappa}$ in the dilute fraction regime (surface mostly reflecting) for arbitrary homogeneous (pores identical) and heterogeneous (pores different) absorbing sets $\Gamma_{a}$. This is attained by solving a cell problem and generalizes previous results for uniform square lattices $[5,16]$ to arbitrary Bravais lattices. A key component of our analysis is detailed knowledge of the periodic Green's function, which is discussed in Appendix A. Let $\mathbf{x}=(\mathbf{y}, z) \in \mathbb{R}^{3}$, where $\mathbf{y}=(x, y) \in \mathbb{R}^{2}$; the periodic Green's function, $G(\mathbf{y}, z)$, satisfies

$$
\begin{gathered}
\Delta G(\mathbf{y}, z)=0 \quad \text { for }(\mathbf{y}, z) \in \Omega, \\
G_{z}(\mathbf{y}, 0)=\sum_{(m, n) \in \mathbb{Z}^{2}} \delta(\mathbf{y}-m \mathbf{p}-n \mathbf{q}), \quad \mathbf{y} \in \partial \Omega, \\
G(\mathbf{y}, z) \sim \frac{z}{|\mathcal{P}|}+\mathcal{O}(1) \quad \text { as } z \rightarrow \infty, \\
G(\mathbf{y}+m \mathbf{p}+n \mathbf{q}, z)=G(\mathbf{y}, z) \quad \text { for }(m, n) \in \mathbb{Z}^{2},(\mathbf{y}, z) \in \Omega .
\end{gathered}
$$

The solution of (1.7) for a square lattice was given in [5] as a lattice sum. In Appendix A, we show how to efficiently numerically evaluate this quantity, which improves on a previous mesh-based approach [16] employed for square lattices and generalizes to arbitrary Bravais lattices. The Green's function consists of a singular piece identical to the half-space Green's function, $g(\mathbf{y}, z)$, and a regular piece, $r(\mathbf{y}, z)$ :

$$
G(\mathbf{y}, z)=g(\mathbf{y}, z)+r(\mathbf{y}, z), \quad g(\mathbf{y}, z)=-\frac{1}{2 \pi} \frac{1}{|\mathbf{x}|}
$$

We further define the constant $\bar{R}=r(\mathbf{0}, 0)$, the value of the regular part of the Green's function at the origin.

In the case where the primitive cell $\mathcal{P}$ has $N$ pores of common capacitance $\varepsilon \bar{c}$ and 
locations $\left\{\mathbf{y}_{j}\right\}_{j=1}^{N}$, we find that as $\varepsilon \rightarrow 0$,

$$
\begin{aligned}
& \bar{\kappa} \sim \frac{2 \pi N \varepsilon \bar{c}}{|\mathcal{P}|}\left[1-\frac{2 \pi \varepsilon \bar{c}}{N} \mathcal{F}\left(\mathbf{y}_{1}, \ldots, \mathbf{y}_{N}\right)\right]^{-1}, \\
& \mathcal{F}\left(\mathbf{y}_{1}, \ldots, \mathbf{y}_{N}\right)=N \bar{R}+\sum_{\substack{i=1 \\
j}}^{N} \sum_{\substack{j=1 \\
j \neq i}}^{N} G\left(\mathbf{y}_{i}-\mathbf{y}_{j}, 0\right) .
\end{aligned}
$$

The capacitance of an absorber is a scalar quantity determined solely by its geometry, which characterizes its capture rate. For a precise definition of the capacitance for a single pore, see (5.9). The leading-order term in (1.9) reflects the capacitance per unit area of the primitive cell $\mathcal{P}$. The correction, $\mathcal{F}$, captures the correction due to self $[\bar{R}]$ and pairwise $\left[G\left(\mathbf{y}_{i}-\mathbf{y}_{j}, 0\right)\right]$ interactions.

In section 6 , we show a variety of numerical results of the theory. The validity of the asymptotic formula (1.9) and the numerical evaluation of the Green's function (1.7) is validated against the KMC method on a number of examples. For homogeneous pore configurations, the asymptotic result (1.9) further reduces to

$$
\bar{\kappa} \sim \frac{2 \pi \varepsilon \bar{c}}{|\mathcal{P}|}[1-2 \pi \varepsilon \bar{c} \bar{R}(\mathbf{p}, \mathbf{q})]^{-1},
$$

where we have called out the dependence of the regular part of the Green's function evaluated at the pore on the lattice vectors $\mathbf{p}$ and $\mathbf{q}$ explicitly, $\bar{R}(\mathbf{p}, \mathbf{q})$, to facilitate comparisons between lattices. Among Bravais lattices, the hexagonal lattice maximizes $\bar{R}(\mathbf{p}, \mathbf{q})$ and hence the total capture rate. Finally, in section 7 , we conclude by discussing the results and opportunities for further work.

2. Refining Berg and Purcell: Homogenization and pore-pore interactions. In this section, we give a brief history of results for the fundamental problem of describing the capture rate of patchy and semipermeable surfaces, notably the derivation and use of homogenized boundary conditions. The idea of deriving homogenized PDEs by averaging over regular or random variations at small scales has an extensive literature going back over half a century (see, for example, [28, 37, 38]). In the setting of cellular-scale chemical sensing, this problem was studied by Berg and Purcell in the landmark 1977 paper Physics of Chemoreception [11], which considered the diffusion of particles with diffusivity $D$ and a steady concentration $C(\mathbf{x})$ into a sphere of radius $R$ covered by $N$ well-separated receptors of common radius $a$. The concentration satisfies Laplace's equation,

$$
\begin{gathered}
D \Delta C=0, \quad \mathbf{x} \in \Omega ; \\
C=0 \quad \text { on } \quad \mathbf{x} \in \Gamma_{a} ; \quad D \nabla C \cdot \hat{\mathbf{n}}=0 \quad \text { on } \quad \mathbf{x} \in \Gamma_{r}, ; \\
\lim _{|\mathbf{x}| \rightarrow \infty} C(\mathbf{x})=C_{\infty},
\end{gathered}
$$

where $\Omega$ is the set of points external to the sphere of radius $R, \Gamma_{a}$ is the set of absorbing discs on the surface of the sphere, $\Gamma_{r}$ is the remainder of the sphere's surface which is reflecting, $\hat{\mathbf{n}}$ is the outward-pointing normal on the sphere's surface, and $C_{\infty}$ is the uniform concentration in the far-field. They postulated that the flux of particles into the sphere was

$$
J \approx 4 D N a C_{\infty}
$$


in the dilute pore limit. The sensing rate (i.e., the flux) scales as the perimeter of the receptors, and therefore a cell could have a nearly optimal sensing performance with only a small fraction of surface area receptor coverage, provided the receptors were numerous and distributed over the membrane.

Shoup and Szabo [46] argued that the boundary conditions on the patchy surface could be replaced by a uniform boundary condition of mixed type,

$$
D \nabla C \cdot \hat{\mathbf{n}}=\kappa C,
$$

analogous to an idea proposed earlier by Collins and Kimball [19]. Matching the flux of the Berg-Purcell (BP) formula (2.2) for the sphere yielded

$$
\kappa=\kappa_{\mathrm{bp}}:=\frac{4 D \sigma}{\pi a}, \quad \sigma=\frac{N \pi a^{2}}{4 \pi R^{2}},
$$

where $D$ is the diffusivity of the particle, $a$ is the common receptor radius, and $\sigma$ is the fraction of surface area absorbing. Again, this is valid in the dilute pore limit for which $\sigma \ll 1$. In the modern vernacular, they proposed a homogenized boundary condition for which the detailed pore geometry was replaced with the single parameter, $\kappa$, which matches the flux asymptotically in the dilute pore limit.

Zwanzig [56] further reasoned that the effective $\kappa$ would be a weighted average of the purely absorbing and purely reflecting case giving

$$
\kappa=\kappa_{z w}:=\frac{4 D \sigma}{\pi a(1-\sigma)} .
$$

Although this expression was for finitely many pores on a sphere instead of the periodic planar problem, it is noteworthy in that the limiting behavior of $\kappa$ is correct: as $\sigma \rightarrow 0$, $\kappa \rightarrow 0$, and as $\sigma \rightarrow 1, \kappa \rightarrow \infty$.

These results can be further refined to reflect the clustering of reactive surface sites that are known to play an important biophysical role [21, 43, 52]. For a review of homogenization of the spherical problem, see our previous study [25], which extends these results to incorporate information on the spatial arrangement of receptors. Related capture problems for finite clusters of receptors on the plane have also been considered in several recent studies. In boundary homogenization approaches $[6,7,8]$, the cluster is replaced by a single circular pore on which the Robin boundary condition (1.3) applies, and functional forms of $\kappa$ are postulated and free parameters estimated either numerically or asymptotically. Asymptotic approaches utilize singular perturbation $[12,25]$ or separable methods $[42,50]$ to obtain series approximations of the flux into the absorbing pores.

Homogenizing the capture rate for the problem considered here, namely a planar periodic array of receptors bounding a half-space, presents an additional subtle difficulty. For a spherical target (either partially or fully absorbing) or a finite cluster of absorbing pores on a plane, the probability of capture of a single particle is less than unity and the capture rate is finite; matching this finite capture rate in the homogenized problem determines $\kappa$. For a periodic plane that is either partially or fully absorbing, every diffusing particle is eventually captured, necessitating a different criterion for determining the leakage parameter.

The homogenization philosophy remains much the same: determine an effective leakage parameter $\bar{\kappa}$ for the boundary condition (1.6) that matches the flux into the boundary for particles that originate far from the surface. However, as any steady solution will have a constant flux through each periodic cell into the plane, conservation 
of mass implies a constant flux through every horizontal cross-section of each cell. As such, the analogous steady problem has a linear gradient in the far-field [5, 32]. Once again, it is assumed that transverse variation due to the pore structure decays far above the surface. In nondimensional variables, the steady solution of (1.5) satisfies

$$
\begin{gathered}
\Delta p=0, \quad \mathbf{x} \in \Omega ; \\
p=0 \quad \text { on } \quad \mathbf{x} \in \Gamma_{a} ; \quad \nabla p \cdot \hat{\mathbf{n}}=0 \quad \text { on } \quad \mathbf{x} \in \Gamma_{r}, \\
p(\mathbf{x}) \sim p_{\infty}\left[z+\bar{\kappa}^{-1}\right] \quad \text { as } \quad z \rightarrow \infty
\end{gathered}
$$

where now $p_{\infty}$ is the concentration gradient as $z \rightarrow \infty$. Specifying the gradient, $p_{\infty}$, in the far-field specifies the solution uniquely, and the constant $\bar{\kappa}^{-1}$ is determined by this unique solution [5, 32]. Matching (1.6) in the far-field allows us to identify $\bar{\kappa}$ as the aforementioned leakage parameter.

The change of variables

$$
p(\mathbf{x})=p_{\infty}[z+u(\mathbf{x})]
$$

now yields the homogenization problem [5, 32]

$$
\begin{gathered}
\Delta u(\mathbf{y}, z)=0 \quad \text { for }(\mathbf{y}, z) \in \Omega, \\
u(\mathbf{y}, 0)=0, \quad \mathbf{y} \in \Gamma_{a}, \quad u_{z}(\mathbf{y}, 0)=-1, \quad \mathbf{y} \in \Gamma_{r}, \\
u(\mathbf{y}+m \mathbf{p}+n \mathbf{q}, z)=u(\mathbf{y}, z) \quad \text { for }(m, n) \in \mathbb{Z}^{2}, \quad(\mathbf{y}, z) \in \Omega . \\
\bar{\kappa}^{-1}=\lim _{z \rightarrow \infty} u(\mathbf{y}, z) .
\end{gathered}
$$

Here, the first three conditions (2.6a), (2.6b), (2.6c), together with the condition that $u(\mathbf{y}, z)$ is bounded as $z \rightarrow \infty$, determine a unique solution to the problem (2.6). The leakage parameter $\bar{\kappa}$ is specified by the far-field behavior in (2.6d).

Early work $[23,39]$ made simple geometric approximations of $\bar{\kappa}$. This problem was first considered systematically by Belyaev, Chechkin, and Gadyl'shin [5] for a square cell, who provided variational bounds on $\bar{\kappa}$ and an asymptotic expansion of the leakage parameter in the limit of small pore size. They found for a primitive cell of unit area with a general pore of effective radius $\varepsilon$ that in the diffuse pore limit $(\varepsilon \rightarrow 0)$, asymptotically

$$
\bar{\kappa}^{-1}=\frac{1}{2 \pi \varepsilon \bar{c}}-\bar{R}+A \varepsilon^{2}+\mathcal{O}\left(\varepsilon^{2}\right) .
$$

Here, $\varepsilon \bar{c}$ is the capacitance of the pore, while $\bar{R}$ is the regular part of the periodic Green's function (1.7) which can be expressed in terms of a lattice sum (see Appendix $\mathrm{A}$ and [5]). The constant $A$ is determined by the asymmetry of the pore through its dipole moment and also the regular part of $G$. Compared with the new results of this paper, (1.9), the first two terms of (2.7) are in agreement, while the order $\varepsilon$ term we have reported vanishes in (2.7) due to the homogeneity of the pore configuration considered in [5].

Bruna, Chapman, and Ramon [16] derived the expression (using our nondimensionalization)

$$
\bar{\kappa} \sim \frac{4 a}{1-4 a \bar{R}}
$$


for a square lattice with circular pores of radius $a$ separated by unit distance. Expression (2.8) is a particular case of (1.10) utilizing the fact that the capacitance $\varepsilon \bar{c}=2 a / \pi$ for a circular pore (see (5.10) for details). In [16], a discretization method was used to estimate that $\bar{R} \approx 0.6207$ via a numerical solution to the elliptic problem (1.7). Appendix A describes a new, simple, rapid, and accurate method of evaluating the Green's function (1.7) for general Bravais lattices via lattice sums akin to those introduced in [5].

A major focus of this paper is to determine the efficacy of using homogenized boundary conditions for approximating the capture distribution for the full timedependent problem of a half-space bounded by a plane with a periodic lattice of absorbing pores. A series of papers $[4,9,10,32]$ approximate the solution to this problem numerically by considering a thick but finite layer of height $\ell$ bounded by a reflecting boundary above and a periodic lattice of absorbing pores below. The advantage of this problem is that the mean capture time is finite and scales as $\ell / \bar{\kappa}$ for $\ell \gg 1$. These studies use a variety of Monte Carlo methods to estimate the mean capture time. To better model the spatial configuration of periodic arrays of receptors on a plane bounding a half-space, the functional forms $[9,10]$

$$
\kappa=\kappa_{\mathrm{be}}:=\frac{4 D \sigma}{\pi a} f(\sigma), \quad f(\sigma)=\frac{1+A \sqrt{\sigma}-B \sigma^{2}}{(1-\sigma)^{2}}
$$

have been proposed. The values of the free parameters were estimated by Monte Carlo particle simulations to be $A=1.49,1.02$ and $B=0.92,0.46$ for pores arranged in hexagonal and square lattices, respectively. These results were further confirmed in [32] by solving the elliptic problem (2.6) for these lattices. We compare these formulas with our numerical results in section 6.1.

A fascinating result, obtained from a complex variables method, is an exact solution of (2.6) by Moizhes [30] for the case of a plane patterned by absorbing infinite stripes (also Appendix A of [32]). This gives

$$
\bar{\kappa}(\sigma)=-\frac{\pi \sigma}{2 \ln \sin \frac{\pi \sigma}{2}},
$$

where $\sigma$ is the absorbing surface area fraction of the plane. In section 4.3, this formula provides a valuable benchmark for evaluating the accuracy of our numerical methods (an idea introduced and employed in [32]).

3. Kinetic Monte Carlo. Monte Carlo simulations provide a valuable tool for numerically estimating the distribution of capture times of diffusing particles for problems such as (1.1) and have been used extensively [4, 6, 7, 8, 9, 10, 27, 33, 34]. In its simplest form, a Monte Carlo method simulates the diffusive (Brownian) motion of a particle as a sequence of small displacements of randomly chosen orientation which terminates when the particle transits an absorbing surface. The algorithm is repeated for many particles (millions or even billions) to sample the capture time distribution. These Monte Carlo methods are hampered by a set of problems. First, the adoption of a fixed stepsize introduces an error at that lengthscale. Second, in capture problems such as (1.1) with fat-tailed distributions a significant fraction of realizations undergo long excursions before they are captured, particularly when the domain is unbounded or the pores are small.

To mitigate such difficulties, Northrup [33] precomputed certain Brownian paths away from the target surface and validated the original BP formula (2.2). Batsilas, 
Berezhkovskii, and Shvartsman [4] used two propagators: the first would move particles to the surface of a sphere contained in the bulk, and the second would estimate capture probabilities within a boundary layer at the target surface. These ideas were precursors to an efficient strategy for these diffusion problems called kinetic Monte Carlo (KMC). In KMC, the diffusion process is broken into steps, where each step corresponds to a diffusion problem on a simpler geometry that can be solved analytically. For example, in our case of a reflecting plane with periodic absorbing pores, the distribution of the time and location of the first impact on the plane can be solved analytically and numerically sampled, replacing the simulation of long excursions of the particles with a single calculation. Early work used these ideas in $N$-body simulations of kinetic gases [34] and chemical reactions [55].

We harness these ideas to produce an effective simulation for sampling the capture time distribution of particles executing Brownian motion in a half-space bounded by a patterned plane consisting of reflecting and absorbing regions. Consider a particle initially located above the plane and undergoing diffusion. It will eventually strike the plane, perhaps repeatedly, until it alights upon the absorbing set. We break this excursion into an alternating sequence of two stages:

- Stage I: Projection from bulk to the plane. Starting from the bulk, the particle is propagated forward to the first impact on the plane. The location and time are drawn from exact distributions given in section 3.1. If the particle impacts an absorbing section, the time is recorded and the algorithm halts. Otherwise, proceed to Stage II.

- Stage II: Projection from plane to the bulk. Calculate the shortest distance $R$ to the absorbing set, and propagate the particle forward to a random location on a hemisphere with radius $R$. The arrival time to the hemisphere is drawn from a second known exact distribution, given in section 3.2. Next, repeat Stage I.

This process is illustrated in Figure 3. For a particular realization, the method alternates between Stages I and II until the particle encounters an absorbing portion of the plane. The first stage of this method replaces the long transients in the bulk (due to the fat-tailed capture time distribution) with a single step. Berg and Purcell [11] described Stage II as a diffusing molecule that has bumped against the surface of a cell ... most likely hitting the cell many times before it wanders away for good. In fact, for Brownian motion, the particle will strike the surface (almost certainly) infinitely often before it departs (which is clear from the idea of a Brownian bridge [20]). Our sampling strategy for Stage II again replaces this with a single step.

This method can be applied to pores of general geometry; circles and stripe geometries are simplest, as the calculation of the signed distance to the attracting set $\Gamma_{a}$ is straightforward. Let us now examine the details of the two stages described above.

3.1. KMC Stage I: The joint distribution to the plane. Consider a particle initially $(t=0)$ at a point $\mathbf{x}_{0}=\left(0,0, z_{0}\right)$. The arrival time and position distribution for the particle's first impact with the plane can be computed by solving for the density $p(x, y, z, t)$, where

$$
\begin{aligned}
p_{t} & =\Delta p, \quad z>0, \quad t>0 ; \\
p(x, y, z, 0) & =\delta(x) \delta(y) \delta\left(z-z_{0}\right), \quad z>0 \\
p(x, y, 0, t) & =0, \quad t>0 .
\end{aligned}
$$




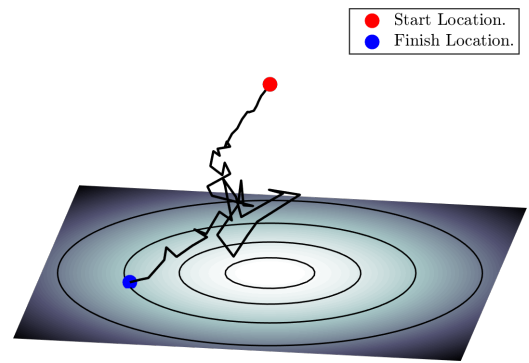

(a) Stage I: Projection from bulk to plane.

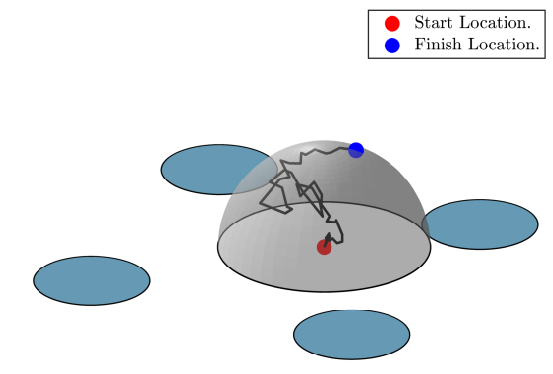

(b) Stage II: Projection from plane to bulk.

FIG. 3. The two stages in propagating a particle forward with the KMC method.

The solution of (3.1), determined from the method of images and the known free space Green's function, is

$$
p(x, y, z, t)=\frac{1}{(4 \pi t)^{3 / 2}}\left[e^{-\frac{x^{2}+y^{2}+\left(z-z_{0}\right)^{2}}{4 t}}-e^{-\frac{x^{2}+y^{2}+\left(z+z_{0}\right)^{2}}{4 t}}\right] .
$$

The flux density through the boundary, $\rho_{T}(t)$, is the probability distribution of transit times to the plane:

$$
\rho_{T}(t)=\int_{x=-\infty}^{\infty} \int_{y=-\infty}^{\infty} p_{z}(x, y, 0, t) d x d y=\frac{z_{0}}{2 \sqrt{\pi} t^{3 / 2}} e^{-\frac{z_{0}^{2}}{4 t}} .
$$

The associated cumulative distribution is given by

$$
P_{T}(t)=\int_{0}^{t} \rho_{T}(\omega) d \omega=\int_{0}^{t} \frac{z_{0}}{2 \sqrt{\pi} \omega^{3 / 2}} e^{-\frac{z_{0}^{2}}{4 \omega}} d \omega=\operatorname{erfc}\left(\frac{z_{0}}{2 \sqrt{t}}\right) .
$$

To sample the transit time distribution, we take a uniform random number $\xi \in[0,1]$ and calculate

$$
t_{*}=\frac{1}{4}\left[\frac{z_{0}}{\operatorname{erfc}^{-1}(\xi)}\right]^{2} .
$$

The spatial distribution of particles at the arrival time $t_{*}, \rho_{X Y}\left(x_{*}, y_{*}\right)$, can now be computed as

$$
\begin{aligned}
\rho_{X Y}\left(x_{*}, y_{*}\right) \equiv \frac{p_{z}\left(x_{*}, y_{*}, 0, t_{*}\right)}{\rho_{T}\left(t_{*}\right)} & =\frac{1}{4 \pi t_{*}} e^{-\frac{x_{*}^{2}+y_{*}^{2}}{4 t_{*}}} \\
& =\left[\frac{1}{\sqrt{4 \pi t_{*}}} e^{-\frac{x_{*}^{2}}{4 t_{*}}}\right]\left[\frac{1}{\sqrt{4 \pi t_{*}}} e^{-\frac{y_{*}^{2}}{4 t_{*}}}\right]=\rho_{X}\left(x_{*}\right) \rho_{Y}\left(y_{*}\right),
\end{aligned}
$$

which is the product of two Gaussian (normal) distributions. Accordingly, $x_{*}$ and $y_{*}$ are both drawn from $\mathcal{N}\left(0,2 t_{*}\right)$.

To summarize, one first determines the time that elapses to impact, $t_{*}$, via (3.5), followed by the horizontal displacements, $x_{*}$ and $y_{*}$, via (3.6). Finally, a check is made to see whether the particle has been captured by landing in the absorbing set on the surface. 
3.2. Stage II: The joint distribution on the sphere. If the particle has impacted a reflecting portion of the surface, we next propagate it forward to the surface of a hemisphere of radius $R$ centered at the impact point. The value of $R$ is chosen as the radius of the largest circle that can be inscribed on the surface that remains within the reflecting portion (i.e., the minimum distance to a point in the absorbing set). The joint distribution for the first exit time and exit location on the hemisphere can be deduced by noting that the equivalent problem on the full sphere has a solution that is radial, thus ensuring that the reflection symmetry on the surface is satisfied. We wish to solve

$$
\begin{aligned}
p_{t} & =\Delta p, \quad z>0, \quad x^{2}+y^{2}+z^{2}<R^{2}, \quad t>0 ; \\
p(x, y, z, 0) & =\delta(x) \delta(y) \delta\left(z-0^{+}\right), \quad z>0, \quad x^{2}+y^{2}+z^{2}<R^{2} ; \\
p(x, y, z, t) & =0, \quad x^{2}+y^{2}+z^{2}=R^{2}, \quad z>0, \quad t>0 ; \\
p_{z}(x, y, 0, t) & =0, \quad x^{2}+y^{2}<R^{2}, \quad t>0,
\end{aligned}
$$

where it is understood that the $\delta$ function is just above the bottom surface. The density distribution is radial, $p(x, y, z, t)=p(r, t)$ for $r=\sqrt{x^{2}+y^{2}+z^{2}}$, and has a separation of variables solution (cf. [27]):

$$
p(r, t)=\sum_{n=1}^{\infty} \frac{n}{R^{2}} \frac{\sin (n \pi r / R)}{r} e^{-n^{2} \pi^{2} t / R^{2}}, \quad t>0 .
$$

The series converges slowly for small $t$ due to the $\delta$-function initial condition; we will show how to ameliorate this difficulty presently. The exit flux density can be computed by integrating over the hemisphere:

$$
\rho_{S}(t)=-\iint_{S} \nabla p \cdot \hat{n} d S=-2 \pi R^{2} p_{r}(R, t)=2 \sum_{n=1}^{\infty}(-1)^{n+1} \frac{n^{2} \pi^{2}}{R^{2}} e^{-n^{2} \pi^{2} t / R^{2}} .
$$

This in turn yields the cumulative exit distribution

$$
P_{S}(t)=1-\int_{\omega=t}^{\infty} \rho_{S}(\omega) d \omega=1+2 \sum_{n=1}^{\infty}(-1)^{n} e^{-n^{2} \tau}, \quad \tau=\frac{\pi^{2} t}{R^{2}}
$$

Here, we have used the total flux (unity) minus the flux at larger times so as to integrate the flux density where it has strong convergence. The expression (3.8) converges quickly for large $t$, but for smaller $t$ values, many terms may be required. However, a theta function identity (that can be derived via the Poisson summation formula) can be used to remedy this issue (cf. [49, Chap. 4]):

$$
\sum_{n=-\infty}^{\infty} e^{-\pi q(n+a)^{2}}=\sum_{n=-\infty}^{\infty} q^{-1 / 2} e^{-\pi n^{2} / q} e^{2 \pi i n a}
$$

Applying the identity (3.9), with $a=\frac{1}{2}$ and $q=\pi / \tau$, to (3.8) yields that

$$
P_{S}(t)=2 \sqrt{\frac{\pi}{\tau}} \sum_{n=0}^{\infty} e^{-\pi^{2}\left(n+\frac{1}{2}\right)^{2} / \tau}, \quad \tau=\frac{\pi^{2} t}{R^{2}} .
$$

The cumulative exit distribution is shown in Figure 4. 


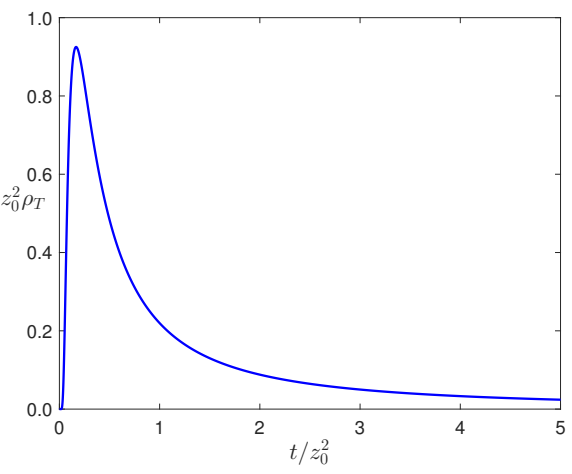

(a) PDF to plane

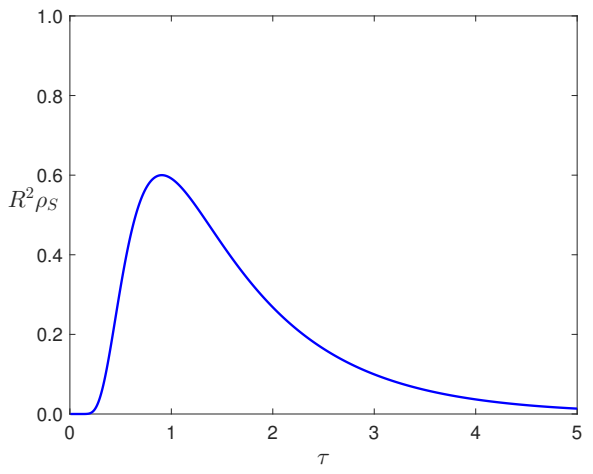

(b) PDF to sphere

FIG. 4. The transit time distributions to the plane and hemisphere. At small times, both distributions are exponentially flat. At large times, the tail of the distribution decays exponentially for the sphere but algebraically (as $t^{-3 / 2}$ ) for the plane due to long excursions into the unbounded half-space.

To sample an arrival time to the sphere, a uniform random number $\xi \in(0,1)$ is chosen and the equation

$$
P_{S}\left(\tau_{*}\right)=\xi
$$

is solved numerically for $\tau_{*}$ that in turn yields the exit time $t_{*}=R^{2} \tau_{*} / \pi^{2}$. The values of $P_{S}(\tau)$ are precomputed and tabulated for computational efficiency (using (3.8) for $\tau \geq 1$ and (3.10) for $\tau<1$ ), and the value of $\tau_{*}$ is determined by linear interpolation, unless $\xi$ is close to unity, in which case an asymptotic approximation is used. Once an exit time has been determined, an exit point on the sphere must be chosen isotropically. As the surface area element satisfies

$$
d S=R^{2} \sin \phi d \theta d \phi=R d \theta d z,
$$

one can draw two random numbers $(\xi, \zeta)$ uniformly in $[0,1]^{2}$ and then choose an exit point that is displaced from the sphere's center by an amount

$$
\left(x_{*}, y_{*}, z_{*}\right)=R\left(\sqrt{1-\zeta^{2}} \cos 2 \pi \xi, \sqrt{1-\zeta^{2}} \sin 2 \pi \xi, \zeta\right) .
$$

As the exit point will be within the bulk, one next returns to Stage I of the KMC method.

More details of our KMC can be found in [45]. In section 4.3, we validate the method for the exactly solvable case of absorbing stripes.

4. Homogenization. As discussed in the introduction to this paper, the idea of homogenization has a long and rich history. Our approach to this problem strongly leverages two previous studies [5, 32], which study homogenization of absorption by a periodically patterned planar boundary (1.5) yielding the Robin boundary condition (1.6). The philosophy in both these papers is to choose $\bar{\kappa}$ so that the capture flux into the bottom boundary exactly matches the unhomogenized boundary condition.

Belyaev, Chechkin, and Gadyl'shin [5] studied the static problem of determining the leakage parameter, $\bar{\kappa}$, for (2.6), specialized to a square lattice of pores. They averaged the problem in horizontal layers; if one defines the horizontal average,

$$
\bar{u}(z) \equiv \iint_{\mathcal{P}} u(\mathbf{y}, z) d \mathbf{y}
$$


then it satisfies $\bar{u}_{z z}=0$ in the bulk, which together with the far-field boundary condition (2.6d) implies $\bar{u}(z)=\bar{\kappa}^{-1}$, where $\bar{\kappa}^{-1}$ is to be determined. The leakage parameter was determined by averaging the Robin boundary condition (1.6) over the boundary at $z=0$ which yields

$$
\begin{gathered}
\iint_{\mathcal{P}} p_{z}(\mathbf{y}, 0) d \mathbf{y}=\iint_{\mathcal{P}} \bar{\kappa} p(\mathbf{y}, 0) d \mathbf{y} \\
\iint_{\mathcal{P}} p_{\infty}[u(\mathbf{y}, 0)+z]_{z} d \mathbf{y}=\bar{\kappa} \iint_{\mathcal{P}} p_{\infty} u(\mathbf{y}, 0) d \mathbf{y} \\
\iint_{\mathcal{P}} 1 d \mathbf{y}=\bar{\kappa} \iint_{\mathcal{P}} u(\mathbf{y}, 0) d \mathbf{y}
\end{gathered}
$$

where we remind the reader that (i) $p(\mathbf{y}, z)=p_{\infty}[u(\mathbf{y}, 0)+z]$, and (ii) the vertical flux of $p(\mathbf{y}, z)$ through each cell is constant and the far-field boundary condition (2.6d) guarantees that the vertical flux of $u(\mathbf{y}, z)$ vanishes. This can be rewritten as

$$
\bar{\kappa}^{-1} \equiv \frac{1}{|\mathcal{P}|} \iint_{\mathcal{P}} u(\mathbf{y}, 0) d \mathbf{y} ;
$$

that is, the leakage parameter is the inverse of the average of $u(\mathbf{y}, 0)$ over a cell at the planar boundary. Finally, they performed matched asymptotics in the small pore limit to approximate $\bar{\kappa}$, which they find scales as the pore radius, a result that is recognizable as BP scaling. Equation (4.1) implies that the total flux (which is unity) is proportional to the leakage parameter times the average concentration on the boundary. As the leakage parameter $\bar{\kappa}$ scales as the pore radius, this implies the concentration on the boundary scales as the inverse of the pore radius. An interpretation of this is that a large number of particles must accumulate on the boundary to achieve unit flux through the small pores.

Their analysis (see also section A.3) allows one to use separation of variables ideas to estimate that the horizontal variation in the full solution to (1.5) is restricted to a boundary layer and decays as $e^{-z / \lambda}$, where $\lambda=2 \pi \max \{|\mathbf{p}|,|\mathbf{q}|\}$. This result justifies the homogenization for a sufficiently deep layer; the simple homogenized boundary condition (1.6) reproduces the asymptotics of the static solution far above the periodically patterned surface, at least for the static problem (2.5).

Muratov and Shvartsman [32] studied the dynamic problem (1.5) in a domain of finite vertical extent, $0<z<\ell$, with a reflecting upper boundary. If one homogenizes the problem by averaging horizontally across the domain and then starts a particle on the bottom surface, the mean capture time for a particle in the homogenized equation is exactly $\ell / \bar{\kappa}$. This allows one to use Monte Carlo simulations to estimate the mean capture time and determine $\bar{\kappa}$, a strategy also used in $[4,9,10]$. The authors solved the static problem (2.6) numerically for $\bar{\kappa}$ via an optimal multigrid approach. They argue that as the mean time to capture is long, $\ell / \bar{\kappa} \gg 1$, whenever $\bar{\kappa}$ is small (which is the small pore limit) or the box height is large, the boundary layer relaxes to the steady solution and the particle spends most its time above the boundary layer where the transverse variation due to the pore structure is exponentially small.

The difficulty of adopting the dynamic approach of [32] to the present problem is that our domain is infinite, and although every particle will eventually be captured by the absorbing portions of the surface, the mean capture time is unbounded (cf. (1.2) and section 4.1), which negates its use as a metric for determining $\bar{\kappa}$. Our strategy is to numerically compute the full capture time distribution with the KMC method described above and compare the results to the capture time distribution for the 
homogenized problem obtained analytically, the details of which are described in the remainder of this section. We can further justify the quasi-static approximation (using ideas akin to $[5,32]$ ). The density for a completely absorbing planar surface was derived in (3.2) and shows the capture time distribution, $\rho_{T}(t) \sim \frac{z_{0}}{2 \sqrt{\pi}} t^{-3 / 2}$, for $t \gg 1$, which has infinite mean (cf. (3.3)). Moreover, we can compute the mean height above the layer as a function of time after the release:

$$
\bar{z}(t)=\frac{\iiint_{\Omega} z \rho(x, y, z, t) d \mathbf{x}}{\iiint_{\Omega} \rho(x, y, z, t) d \mathbf{x}}=\frac{z_{0}}{\operatorname{erf}\left(z_{0} /(2 \sqrt{t})\right)} \sim \sqrt{\pi t} \quad \text { for } \quad t \gg z_{0}^{2} .
$$

We see that $\bar{z}(t)$ increases monotonically from $z_{0}$. As such, the particle spends most of its time above the boundary layer (where horizontal variations in the density function are exponentially small), and the flux into the surface is slowly varying (as $t^{-3 / 2}$ for large $t$ ), which validates our quasi-static approximation, in which we replace the diffusion equation with Laplace's equation in the boundary layer.

4.1. Capture distribution for the homogenized problem. In this section, we derive the particle density, $p(z, t)$, for the homogenized version of (1.5) with the homogenized boundary condition (1.6),

$$
\begin{gathered}
\frac{\partial p}{\partial t}=p_{z z}, \quad z>0, \quad t>0 ; \quad p(z, 0)=\delta\left(z-z_{0}\right), \quad z>0 ; \\
p_{z}=\bar{\kappa} p \quad \text { on } \quad z=0,
\end{gathered}
$$

where we assume the particle is released from a height $z=z_{0}$. Carslaw and Jaeger [17] provide a change of variables method to obtain an exact solution for this problem:

$$
p(z, t)=\frac{e^{-\frac{\left(z-z_{0}\right)^{2}}{4 t}}+e^{-\frac{\left(z+z_{0}\right)^{2}}{4 t}}}{2 \sqrt{\pi t}}-\bar{\kappa} e^{\bar{\kappa}\left(\bar{\kappa} t+z+z_{0}\right)} \operatorname{erfc}\left(\frac{2 \bar{\kappa} t+z+z_{0}}{2 \sqrt{t}}\right) .
$$

Our numerics compare the entire capture distribution for the full problem (1.5) and the homogenized problem (4.3), whose solution we have computed above (4.4). The capture rate is given by the flux into the surface

$$
\rho(t ; \bar{\kappa})=p_{z}(0, t)=\frac{\bar{\kappa}}{\sqrt{\pi t}} e^{-\frac{z_{0}^{2}}{4 t}}-\bar{\kappa}^{2} e^{\bar{\kappa}\left(\bar{\kappa} t+z_{0}\right)} \operatorname{erfc}\left(\frac{2 \bar{\kappa} t+z_{0}}{2 \sqrt{t}}\right),
$$

which for large $t$ still decays as $t^{-3 / 2}$, specifically

$$
\rho(t ; \bar{\kappa}) \sim \frac{t^{-3 / 2}}{2 \sqrt{\pi}}\left[z_{0}+\frac{1}{\bar{\kappa}}\right] \quad \text { as } t \rightarrow \infty .
$$

For large $\bar{\kappa}$, the capture rate can be asymptotically expanded as

$$
\rho(t ; \bar{\kappa}) \sim \frac{t^{-3 / 2}}{2 \sqrt{\pi}} z_{0} e^{-\frac{z_{0}^{2}}{4 t}}+\frac{1}{\bar{\kappa}}\left[\frac{t^{-3 / 2}}{2 \sqrt{\pi}}-\frac{t^{-5 / 2}}{4 \sqrt{\pi}} z_{0}^{2}\right] e^{-\frac{z_{0}^{2}}{4 t}}+\mathcal{O}\left(\frac{1}{\bar{\kappa}^{2}}\right) \quad \text { as } \bar{\kappa} \rightarrow \infty
$$

This expression recovers the capture rate for a completely absorbing plane in the infinite $\bar{\kappa}$ limit. Moreover, it foreshadows the difficulty of numerically fitting this expression to our KMC data for large $\bar{\kappa}$, as the difference between the capture rate distributions is $\mathcal{O}(1 / \bar{\kappa})$. 
From the capture rate, we can derive the cumulative flux distribution:

$$
P(t ; \bar{\kappa})=\int_{0}^{t} \rho(\omega ; \bar{\kappa}) d \omega=\operatorname{erfc}\left(\frac{z_{0}}{2 \sqrt{t}}\right)-e^{\bar{\kappa}\left(\bar{\kappa} t+z_{0}\right)} \operatorname{erfc}\left(\frac{2 \bar{\kappa} t+z_{0}}{2 \sqrt{t}}\right) .
$$

We compare this homogenized distribution to our KMC simulations of the capture time distribution.

4.2. Fitting $\bar{\kappa}$ for empirical data. With these distributions in hand, we can simulate the capture of a large number of particles and then try to choose the value of $\bar{\kappa}$ that best fits the data. To do this, we construct the empirical cumulative distribution, $P_{e}$, which measures the fraction of particles captured as a function of $t$. We reorder the particle capture times, $t_{j}$, so that $t_{1}<t_{2}<\cdots<t_{N}$ and define

$$
P_{e}\left(t_{j}\right)=\frac{j-\frac{1}{2}}{N}
$$

which counts the fraction of particles arriving before $t_{j}$ with the particle arriving at $t_{j}$ being counted as half. The function can be extended to intermediate times via linear interpolation. Next, we define a mean absolute error

$$
\mathcal{E}(\bar{\kappa})=\frac{1}{N} \sum_{j=1}^{N}\left|P\left(t_{j} ; \bar{\kappa}\right)-P_{e}\left(t_{j}\right)\right|
$$

and search numerically for the value of $\bar{\kappa}$ that minimizes the error. This sum effectively weights the error by the flux density and converges at large $N$ to the absolute error

$$
\mathcal{E}(\bar{\kappa}) \sim \int_{\mu=0}^{1}\left|P(t ; \bar{\kappa})-P_{e}(t)\right| d \mu
$$

where $t=P_{e}^{-1}(\mu)$.

We have confirmed the results by considering two other error norms. The first is to use a mean square error. The second utilizes maximum likelihood. Specifically, the probability of observing a set of times $t_{i}$ given a particular $\bar{\kappa}$ is given by $\prod_{i=1}^{N} \rho\left(t_{i} ; \bar{\kappa}\right)$; we can maximize this probability by maximizing

$$
\mathcal{M}(\bar{\kappa})=\sum_{i=1}^{N} \log \rho\left(t_{i} ; \bar{\kappa}\right)
$$

over $\bar{\kappa}$. Thus, this $\bar{\kappa}$ is the most likely.

Comparing the three objective functions gives a check on consistency in computing $\bar{\kappa}$. In practice, all three results agree well when using $10^{6}$ particles and small to moderate values of $\sigma$. For larger $N$, the maximum likelihood approach becomes unstable due to the large number of terms in the sum whose size can vary by several orders of magnitude.

Empirically, we have found that the absolute error is the most stable estimator in the large $N$ limit. As our asymptotics are verified for small to moderate $\sigma$, where the three methods agree strongly, we are confident in the results reported here.

4.3. Homogenization for stripes. To validate the KMC method, we utilize the exact solution (2.10) for $\bar{\kappa}(\sigma)$ derived for the case of infinite absorbing stripes where $\sigma \in(0,1)$ is the proportion of the plane which is absorbing. In Figure 5 , we 


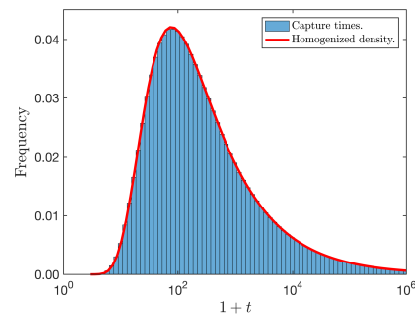

(a) the arrival time distribution

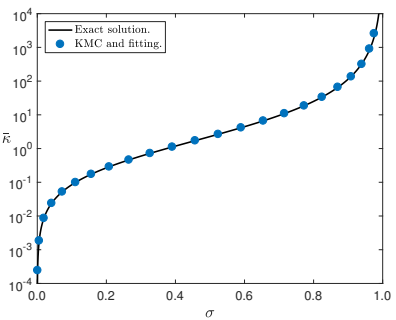

(b) $\bar{\kappa}$ against $\sigma$

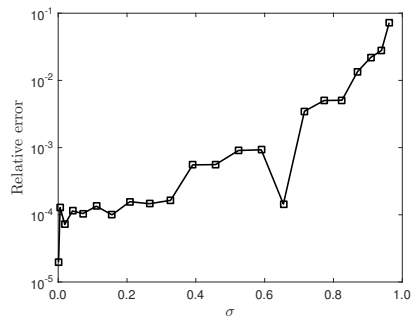

(c) relative error against $\sigma$

FIG. 5. Homogenization for absorbing strips. (a) Frequency of hitting times from the KMC method and the homogenized density (red solid). For an absorbing fraction of $\sigma=0.25$, the value $\bar{\kappa}=0.4092$ is estimated. (b) Agreement between $\bar{\kappa}$ values obtained from the KMC method (blue circles) and the exact (black solid) expression (2.10) over $\sigma \in(0,1)$ using $10^{9}$ realizations. (c) Relative error (4.8) in the approximation of $\bar{\kappa}$ against $\sigma$ shown in panel (b). As the surface becomes mostly absorbing, the error increases as fewer trajectories contact the reflecting set and provide information on $\bar{\kappa}$. Color is available online only.

show results from KMC simulations performed with $1 \times 10^{9}$ realizations, each with initial location $\mathbf{x}_{0}=(0,0,10)$. The homogenized density accurately captures the principal features of the capture time density, particularly its fat tail, for small and moderate $\sigma$.

In Figure 5(c), we show the relative error

$$
\mathcal{E}_{\text {rel }}=\frac{\left|\bar{\kappa}_{\text {exact }}-\bar{\kappa}_{\text {kmc }}\right|}{\bar{\kappa}_{\text {exact }}}
$$

and observe that the method performs well over a large range of $\sigma$. For values of $\sigma$ close to unity (which are associated with larger $\bar{\kappa}$ ), significantly more particles (we have used up to $10^{9}$ ) are needed to accurately determine $\bar{\kappa}$. The reason for this is that as $\sigma \rightarrow 1$, the surface becomes completely absorbing and the time distribution to the plane is dominated by particles that never hit the reflecting portion of the surface (and which therefore yield no information distinguishing different surface configurations). Correspondingly, fewer realizations give meaningful information that can distinguish between different $\bar{\kappa}$ values. In Figure 5 , we find that $10^{9}$ particles allow us to accurately resolve values of $\bar{\kappa}$ up to $10^{3}$.

5. Asymptotics in the dilute pore limit. In this section, we develop an asymptotic estimate of the leakage parameter $\bar{\kappa}$ in the dilute pore limit where the plane is largely reflecting. In particular, we develop a singular perturbation solution of the homogenized problem (2.6):

$$
\begin{array}{cl}
\Delta u(\mathbf{y}, z)=0 & \text { for }(\mathbf{y}, z) \in \Omega, \\
u(\mathbf{y}, 0)=0, \quad \mathbf{y} \in \Gamma_{a}, & u_{z}(\mathbf{y}, 0)=-1, \quad \mathbf{y} \in \Gamma_{r}, \\
u(\mathbf{y}+m \mathbf{p}+n \mathbf{q}, z)=u(\mathbf{y}, z) & \text { for } \quad(m, n) \in \mathbb{Z}^{2},(\mathbf{y}, z) \in \Omega .
\end{array}
$$

The leakage parameter then follows from the far-field condition that

$$
\bar{\kappa}^{-1}=\lim _{z \rightarrow \infty} u(\mathbf{y}, z) .
$$

The condition (5.1d) that $u$ is bounded as $z \rightarrow \infty$ will be applied repeatedly to determine an asymptotic series for $\bar{\kappa}$ (or, equivalently, $\bar{\kappa}^{-1}$ ) in the small pore radius 
limit. We consider the absorbing set $\Gamma_{a}$ to be $N$ nonoverlapping absorbing pores in the plane $\partial \Omega$ :

$$
\Gamma_{a}=\bigcup_{k=1}^{N} \mathcal{A}_{k}\left(\frac{\mathbf{y}-\mathbf{y}_{k}}{\varepsilon}\right) .
$$

Here, $\mathcal{A}_{k}$ is the shape of the $k$ th pore and $\varepsilon$ is a scale factor which yields a parameterized family of homothetic shapes centered at $\mathbf{y}_{k}$. Our asymptotic result will be obtained in terms of the capacitances and locations of the individual pores on the plane.

There is a long history of analysis of solutions to elliptic and parabolic equations in the presence of small defects and inclusions. For results focused on diffusion to small targets, we refer the reader to $[18,22,24]$ for strongly localized perturbations results $[53,54]$ and for uniformly valid results $[1,2,29,35]$. An important constituent in the analysis of such problems is detailed knowledge of the underlying Green's function. In the present scenario, we consider the periodic Green's function $G(\mathbf{y}, z)$ satisfying [5]

$$
\begin{gathered}
\Delta G(\mathbf{y}, z)=0 \quad \text { for }(\mathbf{y}, z) \in \Omega, \\
G_{z}(\mathbf{y}, 0)=\sum_{(m, n) \in \mathbb{Z}^{2}} \delta(\mathbf{y}-m \mathbf{p}-n \mathbf{q}), \quad \mathbf{y} \in \partial \Omega, \\
G(\mathbf{y}, z) \sim \frac{z}{|\mathcal{P}|}+\mathcal{O}(1) \quad \text { as } z \rightarrow \infty, \\
G(\mathbf{y}+m \mathbf{p}+n \mathbf{q}, z)=G(\mathbf{y}, z) \quad \text { for }(m, n) \in \mathbb{Z}^{2},(\mathbf{y}, z) \in \Omega .
\end{gathered}
$$

The Green's function consists of a singular piece identical to the half-space Green's function, $g(\mathbf{y}, z)$, and a regular piece, $r(\mathbf{y}, z)$ :

$$
G(\mathbf{y}, z)=g(\mathbf{y}, z)+r(\mathbf{y}, z), \quad g(\mathbf{y}, z)=-\frac{1}{2 \pi} \frac{1}{|\mathbf{x}|} .
$$

We further define the constant $\bar{R}=r(\mathbf{0}, 0)$, the value of the regular part of the Green's function at the origin. In Appendix A, we give an explicit solution of (5.3) for general Bravais lattices in terms of an infinite series (a lattice sum) and demonstrate how to efficiently numerically evaluate this object.

5.1. Asymptotic expansion of $\bar{\kappa}$ in the dilute pore limit. We use the method of matched asymptotic expansion in the limit of small pore size, $\varepsilon \rightarrow 0$, for the solution of (5.1) (see $[12,18,25]$ for related expansions). At each order, we obtain a solvability condition that yields a contribution to the leakage parameter, $\bar{\kappa}$, through the far-field boundary condition (5.1d). We develop an outer solution $u=u(\mathbf{y}, z)$ of the form

$$
u(\mathbf{y}, z)=\frac{u_{0}}{\varepsilon}+u_{1}+\varepsilon u_{2}+\varepsilon^{2} u_{3}+\mathcal{O}\left(\varepsilon^{3}\right),
$$

which is valid except in a neighborhood of the pore that vanishes as $\varepsilon \rightarrow 0$. The functions $u_{j}=u_{j}(\mathbf{y}, z)$ may be singular at the pore and are a superposition of the regular solution (which is just a constant), Green's functions (reflecting the absorbing pores in (5.1b)), and potentially gradients of Green's functions which appear if higherorder terms in the expansion (5.5) are considered. This is a singular perturbation 
problem since the solution to the limiting problem ( $\varepsilon$ decreasing to zero) does not approach the constant solution to the limit problem $(\varepsilon=0)$ which corresponds to no absorbing set. We will discover below that the leading-order term in the expansion, $u_{0} / \varepsilon$, is a constant. Intuitively, it can be understood via the analysis of (4.1). The flux through a small pore can be estimated from the BP scalings as proportional to the product of the radius $\varepsilon$ (or, equivalently, its capacitance) and the size of $u(\mathbf{y}, 0)$ at the pore. As the vertical flux through the cell $\mathcal{P}$ is fixed at unity, this forces $u(\mathbf{y}, 0) \sim 1 / \varepsilon$ for the two estimates of the flux to balance.

Inserting the expansion (5.5) into (5.1) yields the following problems for the $u_{j}$ :

$$
\begin{gathered}
\Delta u_{j}(\mathbf{y}, z)=0 \quad \text { for }(\mathbf{y}, z) \in \Omega, \\
u_{j}(\mathbf{y}+m \mathbf{p}+n \mathbf{q}, z)=u_{j}(\mathbf{y}, z) \quad \text { for }(m, n) \in \mathbb{Z}^{2}, \quad(\mathbf{y}, z) \in \Omega, \\
\partial_{z} u_{j}(\mathbf{y}, 0)=\left\{\begin{array}{rl}
0, & j \neq 1, \\
-1, & j=1,
\end{array} \quad \mathbf{y} \in \partial \Omega, \quad \mathbf{y} \neq \mathbf{y}_{k} .\right.
\end{gathered}
$$

The condition (5.1d) implies the additional constraint

$$
u_{j}(\mathbf{y}, z) \text { bounded as } z \rightarrow \infty .
$$

We now seek an inner solution at each of the $N$ pores in a primitive cell of the periodic lattice. To establish the local behavior of $u_{j}$ in the vicinity of the $k$ th pore, we introduce stretched coordinates

$$
\boldsymbol{\zeta}=(\mathbf{s}, \eta), \quad \mathbf{s}=\frac{\mathbf{y}-\mathbf{y}_{k}}{\varepsilon}, \quad \eta=\frac{z}{\varepsilon} .
$$

The local problem is expanded in a form similar to (5.5):

$$
w(\mathbf{s}, \eta)=\frac{w_{0}}{\varepsilon}+w_{1}+\varepsilon w_{2}+\mathcal{O}\left(\varepsilon^{2}\right),
$$

where the $w_{j}=w_{j}(\mathbf{s}, \eta)$ satisfy the following problems in the upper half-space:

$$
\begin{gathered}
\Delta_{\zeta} w_{j}=0, \quad \eta>0, \quad \mathbf{s} \in \mathbb{R}^{2} ; \\
w_{j}(\mathbf{s}, 0)=0, \quad \mathbf{s} \in \mathcal{A}_{k} ; \quad \partial_{\eta} w_{j}(\mathbf{s}, 0)=\left\{\begin{array}{rl}
0, & j \neq 2, \\
-1, & j=2,
\end{array} \quad \mathbf{s} \notin \mathcal{A}_{k} .\right.
\end{gathered}
$$

Here, the Laplacian, $\Delta_{\boldsymbol{\zeta}}$, is now in the stretched $\boldsymbol{\zeta}$ coordinates. The homogeneous solutions to (5.8) are arbitrary to a multiplicative constant which is eventually determined by matching the far-field behavior with the outer solution.

To solve the family of equations (5.8), we introduce a related problem well known from electrostatics (cf. [48]) of an electrified flat plate of shape $\mathcal{A}_{k}$,

$$
\begin{gathered}
\Delta_{\zeta} \bar{w}=0, \quad \eta>0, \quad \mathbf{s} \in \mathbb{R}^{2} ; \\
\bar{w}(\mathbf{s}, 0)=1, \quad \mathbf{s} \in \mathcal{A}_{k} ; \quad \partial_{\eta} \bar{w}(\mathbf{s}, 0)=0, \quad \mathbf{s} \notin \mathcal{A}_{k} ; \\
\lim _{\rho \rightarrow \infty} \bar{w}(\mathbf{s}, \eta)=0,
\end{gathered}
$$

where $\rho \equiv|\boldsymbol{\zeta}|=\sqrt{|\mathbf{s}|^{2}+\eta^{2}}$ is the distance to the plate's center. The Neumann condition external to the plate corresponds to a symmetry in the $z=0$ plane, while 
the plate itself is held at a constant potential. The homogeneous solution for $w_{k}$ in (5.8) is given by any constant multiple of $1-\bar{w}$.

In terms of the capacitance $c_{k}$ of the shape $\mathcal{A}_{k}$, the far-field behavior (cf. [5]) is

$$
\bar{w} \sim \frac{c_{k}}{\rho}+\mathcal{O}\left(\frac{1}{\rho^{2}}\right)+\cdots \quad \text { as } \quad \rho \rightarrow \infty .
$$

For the case where $\mathcal{A}_{k}$ is circular, $|\mathbf{s}| \leq a_{k}$, (5.9) corresponds to the well-known electrified disk problem (cf. [48]) with solution

$$
\bar{w}(\mathbf{s}, \eta)=\frac{2}{\pi} \sin ^{-1}\left[\frac{2 a_{k}}{\sqrt{\left(|\mathbf{s}|+a_{k}\right)^{2}+\eta^{2}}+\sqrt{\left(|\mathbf{s}|-a_{k}\right)^{2}+\eta^{2}}}\right], \quad c_{k}=\frac{2 a_{k}}{\pi} .
$$

In terms of the electrified disk solution, $\bar{w}$, we find that the leading-order pore solution is

$$
w_{0}=E_{k}(1-\bar{w}(\mathbf{s}, \eta))
$$

where the constant $E_{k}$ is eventually determined by matching with the far-field. We remark that the solution of $w_{0}$ for the $k$ th pore depends on the shape of $\mathcal{A}_{k}$; however, to determine an expansion for $\bar{\kappa}^{-1}$ up to $\mathcal{O}\left(\varepsilon^{2}\right)$, only the capacitance $c_{k}$ for each pore is required. Expressing the far-field behavior in the original coordinates $\mathbf{x}$ provides the following local conditions for $u_{0}$ and $u_{1}$ :

$$
u_{0} \sim E_{k}, \quad u_{1} \sim-\frac{E_{k} c_{k}}{\left|\mathbf{x}-\mathbf{x}_{k}\right|}+\mathcal{O}(1) \quad \text { as } \quad \mathbf{x} \rightarrow \mathbf{x}_{k},
$$

where we denote the location of the $k$ th pore in $\mathbb{R}^{3}$ as $\mathbf{x}_{k}=\left(\mathbf{y}_{k}, 0\right)$. Since $u_{0}$ is bounded at each pore, $u_{0}$ must be the constant solution to (5.6). Therefore, the conditions $u_{0}=E_{k}$ for $k=1, \ldots, N$ imply that the $E_{k}$ terms are equal. The general solution of (5.6) for $j=1$ which incorporates the local condition (5.11) is

$$
u_{1}=-z+2 \pi u_{0} \sum_{k=1}^{N} c_{k} G\left(\mathbf{y}-\mathbf{y}_{k}, z\right)+\chi_{1},
$$

where $\chi_{1}$ is a constant to be determined. The limiting behavior (5.1d) implies that the solvability condition for $u_{1}$ is that the solution be bounded as $z \rightarrow \infty$. Applying this solvability condition to (5.12) with the limiting behavior (5.3c) for $G$ as $z \rightarrow \infty$ gives

$$
u_{0}=\frac{|\mathcal{P}|}{2 \pi N \bar{c}}, \quad \bar{c}=\frac{1}{N} \sum_{k=1}^{N} c_{k} .
$$

To obtain $\chi_{1}$ and fully specify the correction $u_{1}$, we project the local behavior of (5.12) onto each of the pores. Near the $k$ th pore we have that

$$
\frac{u_{0}}{\varepsilon}+u_{1}+\cdots \sim \frac{u_{0}}{\varepsilon}\left(1-\frac{c_{k}}{\left|\mathbf{x}-\mathbf{x}_{k}\right|}\right)+2 \pi u_{0} B_{k}+\chi_{1}+\mathcal{O}(\varepsilon) \quad \text { as } \quad \mathbf{x} \rightarrow \mathbf{x}_{k} .
$$

The pore interaction terms $B_{k}$ are defined as

$$
B_{k}=c_{k} \bar{R}+\sum_{\substack{j=1 \\ j \neq k}}^{N} c_{j} G\left(\mathbf{y}_{k}-\mathbf{y}_{j}, 0\right)
$$


where $G\left(\mathbf{y}_{k}-\mathbf{y}_{j}, 0\right)$ captures the pairwise interaction of the pores at $\mathbf{y}_{k}$ and $\mathbf{y}_{j}$ in a primitive cell of the lattice (and also the interaction with their periodic images, as we are using the periodic Green's function).

A comparison between (5.7) and (5.14) yields the matching condition $\lim _{\rho \rightarrow \infty} w_{1}=$ $\left(2 \pi u_{0} B_{k}+\chi_{1}\right)$, which implies that

$$
w_{1}=\left(2 \pi u_{0} B_{k}+\chi_{1}\right)(1-\bar{w}(\mathbf{y})),
$$

where $\bar{w}(\mathbf{y})$ solves (5.9). The local behavior on $u_{2}$ is then given to be

$$
u_{2} \sim-\frac{c_{k}\left(2 \pi u_{0} B_{k}+\chi_{1}\right)}{\left|\mathbf{x}-\mathbf{x}_{k}\right|}+\cdots \quad \text { as } \quad \mathbf{x} \rightarrow \mathbf{x}_{k} .
$$

The general solution of (5.6) for $j=2$ which incorporates the local condition (5.17) is

$$
u_{2}=2 \pi \sum_{k=1}^{N} c_{k}\left(2 \pi u_{0} B_{k}+\chi_{1}\right) G\left(\mathbf{y}-\mathbf{y}_{k}, z\right)+\chi_{2},
$$

where $\chi_{2}$ is an arbitrary constant. The solvability condition that $u_{2}$ be bounded as $z \rightarrow \infty$ specifies that

$$
\sum_{k=1}^{N} c_{k}\left(2 \pi u_{0} B_{k}+\chi_{1}\right)=2 \pi u_{0} \sum_{k=1}^{N} c_{k} B_{k}+\chi_{1} N \bar{c}=0 .
$$

Rearranging the equality (5.19) for $\chi_{1}$ and applying (5.13) for $u_{0}$ yields that

$$
\chi_{1}=-\frac{2 \pi u_{0}}{N \bar{c}} \mathbf{c}^{T} \mathcal{G} \mathbf{c}
$$

where the matrix $\mathcal{G} \in \mathbb{R}^{N \times N}$ and the vector $\mathbf{c} \in \mathbb{R}^{N}$ are defined as

$$
\mathcal{G}_{i, j}=\left\{\begin{array}{rl}
\bar{R}, & i=j, \\
G\left(\mathbf{y}_{i}-\mathbf{y}_{j}, 0\right), & i \neq j,
\end{array} \quad \mathbf{c}=\left(c_{1}, \ldots, c_{N}\right)^{T} .\right.
$$

To go further and identify the next correction $\chi_{2}$, we write the expansion (5.5) in terms of coordinates near the $k$ th pore and include gradient terms to find that

$$
w_{2}(\boldsymbol{\zeta}) \sim-\eta+2 \pi u_{0} \mathbf{f}_{k} \cdot \mathbf{s}+2 \pi D_{k}+\chi_{2}+\mathcal{O}(1), \quad \rho \rightarrow \infty .
$$

Here, $\mathbf{f}_{k} \in \mathbb{R}^{2}$ is the horizontal gradient term arising from (5.12), while $D_{k}$ arises from the local behavior of (5.18). The gradient terms are given by

$$
\mathbf{f}_{k}=\left[c_{k} \nabla_{\mathbf{y}} R\left(\mathbf{y}-\mathbf{y}_{k}, 0\right)+\sum_{\substack{j=1 \\ j \neq k}}^{N} c_{j} \nabla_{\mathbf{y}} G\left(\mathbf{y}-\mathbf{y}_{k}, 0\right)\right]_{\mathbf{y}=\mathbf{y}_{k}},
$$

while the constant terms $D_{k}$ for $k=1, \ldots, N$ are given by

$$
\begin{aligned}
D_{k} & =c_{k}\left(2 \pi u_{0} B_{k}+\chi_{1}\right) \bar{R}+\sum_{\substack{j=1 \\
j \neq k}}^{N} c_{j}\left(2 \pi u_{0} B_{j}+\chi_{1}\right) G\left(\mathbf{y}_{j}-\mathbf{y}_{k}, 0\right) \\
& =\chi_{1} B_{k}+2 \pi u_{0}\left[c_{k} B_{k} \bar{R}+\sum_{\substack{j=1 \\
j \neq k}}^{N} c_{j} B_{j} G\left(\mathbf{y}_{j}-\mathbf{y}_{k}, 0\right)\right]
\end{aligned}
$$


The general solution of (5.8) for $j=2$ which matches the far-field behavior (5.22) is of the form

$$
w_{2}(\boldsymbol{\zeta})=-\eta+2 \pi u_{0} \mathbf{f}_{k} \cdot \mathbf{w}_{*}(\boldsymbol{\zeta})+\left(2 \pi D_{k}+\chi_{2}\right)(1-\bar{w}(\boldsymbol{\zeta})) .
$$

The vector-valued problem $\mathbf{w}_{*}(\boldsymbol{\zeta}) \in \mathbb{R}^{2}$ is a planar dipole problem and satisfies

$$
\begin{gathered}
\Delta_{\zeta} \mathbf{w}_{*}=\mathbf{0}, \quad \eta>0, \quad \mathbf{s} \in \mathbb{R}^{2} ; \\
\mathbf{w}_{*}=\mathbf{0}, \quad \eta=0, \quad \mathbf{s} \in \mathcal{A}_{k} ; \quad \partial_{\eta} \mathbf{w}_{*}=\mathbf{0}, \quad \eta=0, \quad \mathbf{s} \notin \mathcal{A}_{k} ; \\
\mathbf{w}_{*}=\mathbf{s}+\mathcal{O}(1), \quad \rho \rightarrow \infty .
\end{gathered}
$$

The exact solution for $\mathbf{w}_{*}(\boldsymbol{\zeta})$ in the case of circular pores is determined in Sneddon [48, p. 177], though the particular details are not important in the determination of $\chi_{2}$ since $\mathbf{w}_{*}(\boldsymbol{\zeta})$ does not contribute a monopole to the far-field. Therefore, the solution of (5.25) provides the local condition $u_{3} \sim-c_{k}\left(2 \pi D_{k}+\chi_{2}\right) /\left|\mathbf{x}-\mathbf{x}_{k}\right|$ as $\mathbf{x} \rightarrow \mathbf{x}_{k}$. The condition that $u_{3}$ remain bounded as $z \rightarrow \infty$ is that

$$
0=\sum_{k=1}^{N} c_{k}\left(2 \pi D_{k}+\chi_{2}\right)=2 \pi \sum_{k=1}^{N} c_{k} D_{k}+N \bar{c} \chi_{2} .
$$

Solving for $\chi_{2}$ and using (5.24) for the terms $D_{k}$ yields the concise expression

$$
\chi_{2}=\frac{\chi_{1}^{2}}{u_{0}}-\frac{4 \pi^{2} u_{0}}{N \bar{c}}(\mathcal{G} \mathbf{c})^{T} \operatorname{diag}(\mathbf{c}) \mathcal{G} \mathbf{c} .
$$

Combining (5.13), (5.20), and (5.28) in condition (5.1d) yields the three-term approximation for $\bar{\kappa}^{-1}$,

$$
\begin{aligned}
\bar{\kappa}^{-1} & =\frac{u_{0}}{\varepsilon}+\chi_{1}+\varepsilon \chi_{2}+\mathcal{O}\left(\varepsilon^{2}\right) \\
& =\frac{|\mathcal{P}|}{2 \pi \varepsilon N \bar{c}}\left(1-\frac{2 \pi \varepsilon}{N \bar{c}} \mathbf{c}^{T} \mathcal{G} \mathbf{c}+\frac{4 \pi^{2} \varepsilon^{2}}{N^{2} \bar{c}^{2}}(\mathcal{G} \mathbf{c})^{T} \mathcal{M}(\mathcal{G} \mathbf{c})+\mathcal{O}\left(\varepsilon^{3}\right)\right),
\end{aligned}
$$

where the matrix $\mathcal{M}$, defined as

$$
\mathcal{M} \equiv \mathbf{c c}^{T}-N \bar{c} \operatorname{diag}(\mathbf{c}),
$$

describes the influence of interpore variations in capacitances for the pore set. To summarize, we have derived an expansion for $\bar{\kappa}^{-1}$ in the limit of vanishing pore size,

$$
\begin{aligned}
\bar{\kappa}^{-1} & =\frac{|\mathcal{P}|}{2 \pi \varepsilon N \bar{c}}\left[1-\frac{2 \pi \varepsilon}{N \bar{c}} \mathbf{c}^{T} \mathcal{G} \mathbf{c}+\frac{4 \pi^{2} \varepsilon^{2}}{N^{2} \bar{c}^{2}}(\mathcal{G} \mathbf{c})^{T} \mathcal{M}(\mathcal{G} \mathbf{c})+\mathcal{O}\left(\varepsilon^{3}\right)\right] \\
& =\frac{|\mathcal{P}|}{N \bar{c}}\left[\frac{1}{2 \pi \varepsilon}-\frac{1}{N \bar{c}} \mathbf{c}^{T} \mathcal{G} \mathbf{c}+\frac{2 \pi \varepsilon}{N^{2} \bar{c}^{2}}(\mathcal{G} \mathbf{c})^{T} \mathcal{M}(\mathcal{G} \mathbf{c})+\mathcal{O}\left(\varepsilon^{2}\right)\right],
\end{aligned}
$$

where the matrix $\mathcal{M}$ is defined in (5.30). In comparing (5.31) with previous results obtained by Belyaev, Chechkin, and Gadyl'shin [5], (2.7), we remark that the result (2.7) is derived for a uniform square lattice of which the $\mathcal{O}(\varepsilon)$ term is zero (as is explained in section 5.2). At the next order, the $\mathcal{O}\left(\varepsilon^{2}\right)$ term describes the effect of the pore's dipole and orientation on the capture rate. This term can be determined by a straightforward extension of the asymptotic procedure; see [26], for example. 
5.2. The effect of pore heterogeneity. In practice, one often wishes to make comparisons with $\bar{\kappa}$ (as opposed to $\bar{\kappa}^{-1}$ ). Two expressions for $\bar{\kappa}$ are

$$
\begin{aligned}
\bar{\kappa} & =\frac{2 \pi \varepsilon N \bar{c}}{|\mathcal{P}|}\left[1-\frac{2 \pi \varepsilon}{N \bar{c}} \mathbf{c}^{T} \mathcal{G} \mathbf{c}+\frac{4 \pi^{2} \varepsilon^{2}}{N^{2} \bar{c}^{2}}(\mathcal{G} \mathbf{c})^{T} \mathcal{M}(\mathcal{G} \mathbf{c})+\mathcal{O}\left(\varepsilon^{3}\right)\right]^{-1} \\
& =\frac{2 \pi \varepsilon N \bar{c}}{|\mathcal{P}|}\left[1+\frac{2 \pi \varepsilon}{N \bar{c}} \mathbf{c}^{T} \mathcal{G} \mathbf{c}+\frac{4 \pi^{2} \varepsilon^{2}}{N \bar{c}}(\mathcal{G} \mathbf{c})^{T} \operatorname{diag}(\mathbf{c})(\mathcal{G} \mathbf{c})+\mathcal{O}\left(\varepsilon^{3}\right)\right]
\end{aligned}
$$

where the second is just a re-expansion in $\varepsilon$ of the reciprocal in the first. We find that the first expression often agrees better with our numerics at modest values of $\varepsilon$. We show below that the contribution $(\mathcal{G} \mathbf{c})^{T} \mathcal{M}(\mathcal{G} \mathbf{c})$ to $\bar{\kappa}^{-1}$ in $(5.32)$ vanishes for pore configurations which have identical capacitances. However, the term at the corresponding order does not vanish in the expansion (5.33). Ultimately, the choice here to expand $\bar{\kappa}$ or $\bar{\kappa}^{-1}$ is arbitrary; depending on the original scalings chosen for the governing equations, one can asymptotically derive either expression and then algebraically determine its inverse.

In both (5.32) and (5.33), the $\mathcal{O}(\varepsilon)$ term reflects the $\mathrm{BP}$ approximation to the leakage parameter $(2.3)$ and the $\mathcal{O}\left(\varepsilon^{2}\right)$ term can be either deprecating or enhancing (see section 6.2.1 for an example). The matrix $\mathcal{M}$, defined in (5.30), is negative semidefinite, and the contribution $(\mathcal{G} \mathbf{c})^{T} \mathcal{M}(\mathcal{G} \mathbf{c})$ vanishes for homogeneous pore configurations. Therefore, from the expansion (5.32), we conclude that this term deprecates the capture rate for heterogeneous configurations in the dilute fraction limit.

To show that $\mathcal{M}$ is negative definite, we consider the quadratic form $\mathbf{v}^{T} \mathcal{M} \mathbf{v}$, where $\mathbf{v}$ is an arbitrary vector. We further decompose $\mathbf{v}$ into two components: $\beta \mathbf{b}$, which is a scalar multiple, $\beta$, of the constant vector $\mathbf{b}=[1,1, \ldots, 1]^{T}$; and $\mathbf{a}$, which is orthogonal to the vector of capacitances $\mathbf{c}$. This orthogonality condition allows us to solve for $\beta$ :

$$
\mathbf{v}=\mathbf{a}+\beta \mathbf{b}, \quad \beta=\frac{\mathbf{c}^{T} v}{N \bar{c}} .
$$

We have used the fact that

$$
\mathbf{c}^{T} \mathbf{b}=\sum_{k=1}^{N} c_{k}=N \bar{c} .
$$

Now consider the quadratic form

$$
\begin{aligned}
\mathbf{v}^{T} \mathcal{M} \mathbf{v} & =\mathbf{a}^{T} \mathcal{M} \mathbf{a}+2 \beta \mathbf{a}^{T} \mathcal{M} \mathbf{b}+\beta^{2} \mathbf{b}^{T} \mathcal{M} \mathbf{b} \\
& =-N \bar{c} \mathbf{a}^{T} \operatorname{diag}(\mathbf{c}) \mathbf{a}-2 \beta N \bar{c} \mathbf{a}^{T} \operatorname{diag}(\mathbf{c}) \mathbf{b}+\beta^{2} \mathbf{b}^{T} \mathbf{c c}^{T} \mathbf{b}-\beta^{2} N \bar{c} \mathbf{b}^{T} \operatorname{diag}(\mathbf{c}) \mathbf{b} \\
& =-N \bar{c} \mathbf{a}^{T} \operatorname{diag}(\mathbf{c}) \mathbf{a}-2 \beta N \bar{c} \mathbf{a}^{T} \mathbf{c}+\beta^{2}(N \bar{c})^{2}-\beta^{2}(N \bar{c})^{2} \\
& =-N \bar{c} \mathbf{a}^{T} \operatorname{diag}(\mathbf{c}) \mathbf{a},
\end{aligned}
$$

which we see is negative, unless $\mathbf{a}=0$. So the $\chi_{2}$ contribution to $\bar{\kappa}^{-1}$ is negative, unless $\mathcal{G} \mathbf{c}$ is a multiple of $\mathbf{b}=[1,1, \ldots, 1]^{T}$, in which case it is zero. We remark that the expansion for $\bar{\kappa}$ derived in [5] for a square lattice did not include this term, precisely because a homogeneous pore configuration was considered. Note that the vector $\mathcal{G} \mathbf{c}$ can be thought of as the sum of the effects of a set of pores on a given pore. If the arrangement of pores is homogeneous, then $\mathcal{G} \mathbf{c}$ is a multiple of $\mathbf{b}=[1,1, \ldots, 1]^{T}$ and $\chi_{2}$ vanishes. 


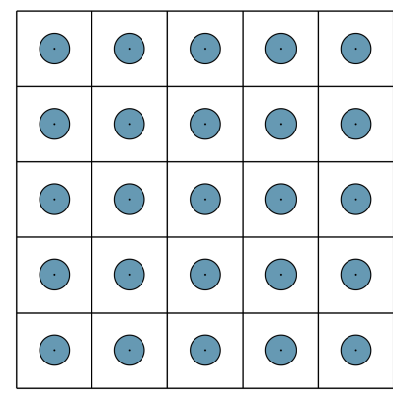

(a) square

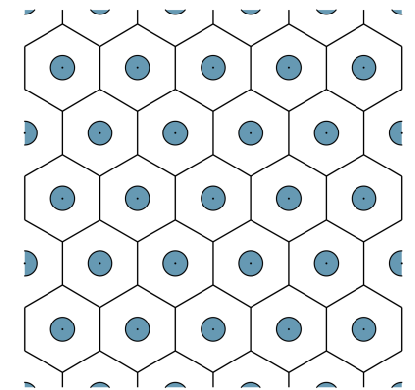

(b) hexagonal

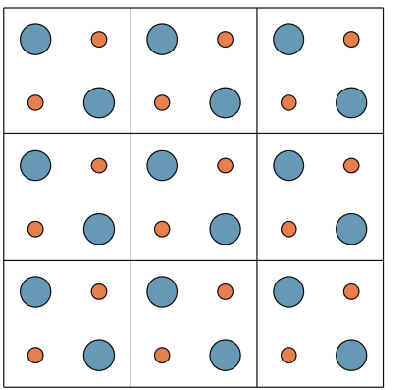

(c) heterogeneous

FIG. 6. Three example lattices used to validate the asymptotic formula.

6. Results. In this section, we perform a number of numerical demonstrations to show the agreement between the KMC method (section 3) and the singular perturbation approach for estimating $\bar{\kappa}$ in the dilute fraction limit (section 5). This agreement also validates the lattice sum evaluation of the Green's function (Appendix A).

6.1. Verification of asymptotic formula. In this section, we verify the validity of the asymptotic formula on three test cases shown in Figure 6. To observe the convergence of the numerical method and validity of the asymptotics, we calculate

$$
\mathcal{E}_{\mathrm{rel}}(\bar{\kappa})=\left|\frac{\bar{\kappa}-\bar{\kappa}_{\mathrm{kmc}}}{\bar{\kappa}_{\mathrm{kmc}}}\right|
$$

where the estimate $\bar{\kappa}_{\mathrm{kmc}}$ is generated from $1 \times 10^{9}$ realizations of the capture time, each initiated at the point $\mathbf{x}_{0}=(0,0,5)$. The asymptotics are benchmarked on three examples: a square, a hexagonal, and a nonuniform lattice (see Figure 6).

6.1.1. Example: Square centered lattice. Here, we compare with the asymptotics for the square centered lattice shown in Figure 6(a) with primitive cell $\mathcal{P}=$ $[-1 / 2,1 / 2]^{2}$ for which $N=1,|\mathcal{P}|=1$, and $\bar{c}=2 / \pi$. Formulating the asymptotic result (5.32) in terms of the absorbing fraction $\sigma=\pi \varepsilon^{2}$ gives

$$
\bar{\kappa}=\frac{4 \sigma}{\pi \varepsilon}\left[\frac{1}{1-4 \sqrt{\frac{\sigma}{\pi}} \bar{R}}+\mathcal{O}\left(\sigma^{\frac{3}{2}}\right)\right] .
$$

The value of the regular part is calculated to be $\bar{R} \approx 0.6207$, in agreement with that determined in [16]. In Figure 7, we show agreement between the estimate, after rescaling by the $\mathrm{BP}$ value (2.3),

$$
\frac{\bar{\kappa}}{\bar{\kappa}_{\mathrm{bp}}}=\left[1-4 \sqrt{\frac{\sigma}{\pi}} \bar{R}\right]^{-1}+\mathcal{O}\left(\sigma^{\frac{3}{2}}\right), \quad \bar{\kappa}_{\mathrm{bp}}=\frac{4 \sigma}{\pi \varepsilon},
$$

and the KMC results for the square lattice. We also compare to the fitted results derived in $[9,10]$. By contrast with the fitted results (which were optimized over a large range of $\sigma$ ), our numerics verify that the asymptotic results capture the behavior of $\bar{\kappa} / \bar{\kappa}_{\mathrm{bp}}$ to $\mathcal{O}\left(\sigma^{\frac{3}{2}}\right)$ in the dilute pore limit. 


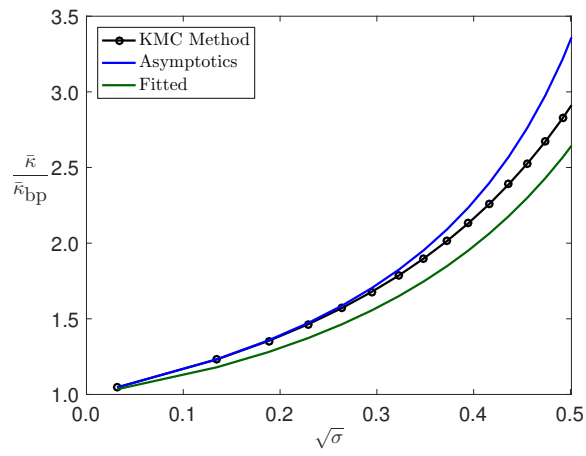

(a) rescaled $\bar{\kappa} / \bar{\kappa}_{\text {bp }}$ against $\sqrt{\sigma}$

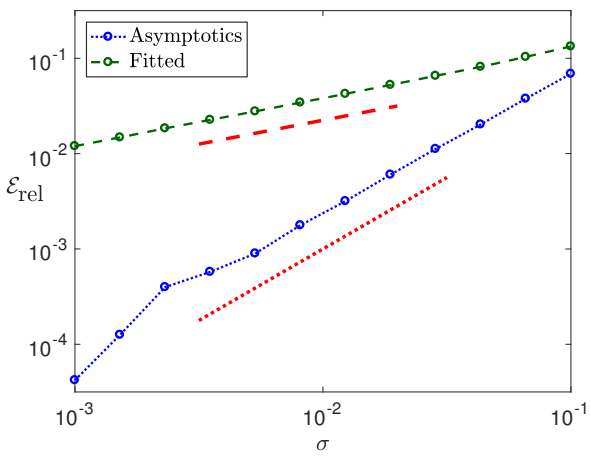

(b) the relative error

FIG. 7. Square centered lattice example. Comparison of the asymptotic formula (blue curves) (6.2) and the fitted results (green curves) (2.9) from [9, 10] with KMC numerics. Left: the rescaled leakage parameter $\bar{\kappa} / \bar{\kappa}_{b p}$ against $\sqrt{\sigma}$. Right: relative error against $\sigma$. Lines (red dashed and dotted) of slope $1 / 2$ and $3 / 2$ added for comparison. Color is available online only.

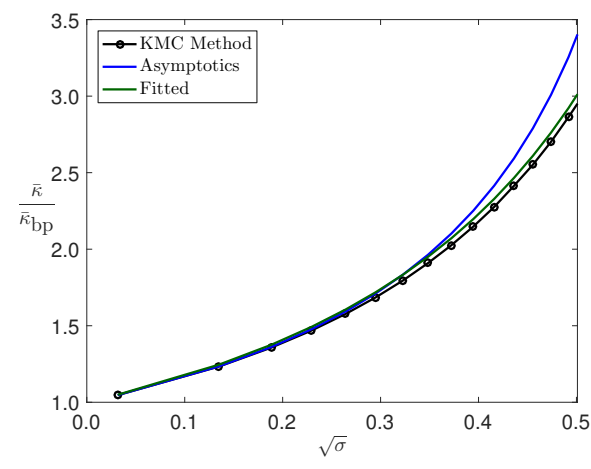

(a) rescaled $\bar{\kappa} / \bar{\kappa}_{\mathrm{bp}}$ against $\sqrt{\sigma}$

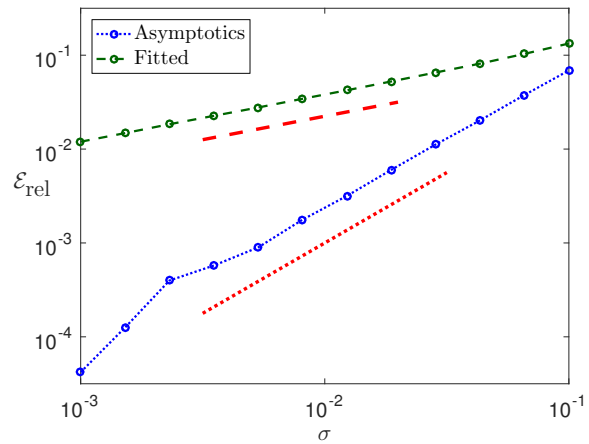

(b) relative errors

FIG. 8. Hexagonal lattice example. Comparison of the asymptotic formula (blue curves) (6.4) and the fitted results (green curves) (2.9) from [9, 10] with KMC numerics. Left: the rescaled leakage parameter $\bar{\kappa} / \bar{\kappa}_{b p}$ against $\sqrt{\sigma}$. Right: relative error against $\sigma$. Lines (red dashed and dotted) of slope $1 / 2$ and $3 / 2$ added for comparison. Color is available online only.

6.1.2. Example: Hexagonal lattice. For the hexagonal lattice with a primitive cell of unit area (cf. Figure $6(\mathrm{~b})$ ), (5.32) is applied with $N=1,|\mathcal{P}|=1$, and $\bar{c}=2 / \pi$ to obtain the rescaled approximation

$$
\frac{\bar{\kappa}}{\bar{\kappa}_{\mathrm{bp}}}=\left[1-4 \sqrt{\frac{\sigma}{\pi}} \bar{R}\right]^{-1}+\mathcal{O}\left(\sigma^{\frac{3}{2}}\right), \quad \bar{\kappa}_{\mathrm{bp}}=\frac{4 \sigma}{\pi \varepsilon} .
$$

We calculate that $\bar{R}=0.6240$. In Figure 8, we display the agreement between (6.4) and the fitted result $(2.9)$ derived in $[9,10]$ with simulations from the KMC method. As with the square lattice, the fitted results are better for moderate $\sigma$, while our numerics verify that the asymptotic results capture the behavior of $\bar{\kappa} / \bar{\kappa}_{\text {bp }}$ to higher order in the dilute pore limit. 


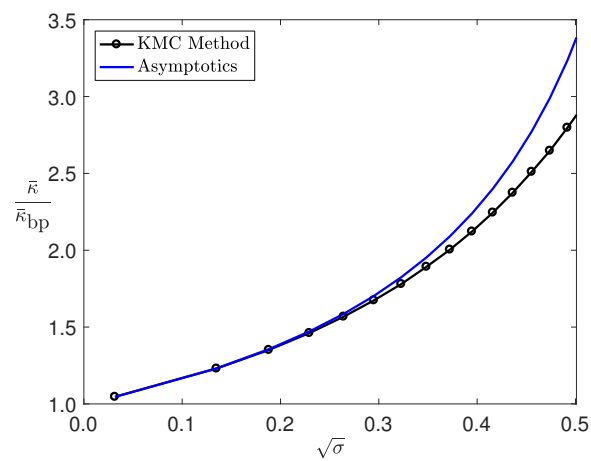

(a) rescaled $\bar{\kappa} / \bar{\kappa}_{\text {bp }}$ against $\sqrt{\sigma}$

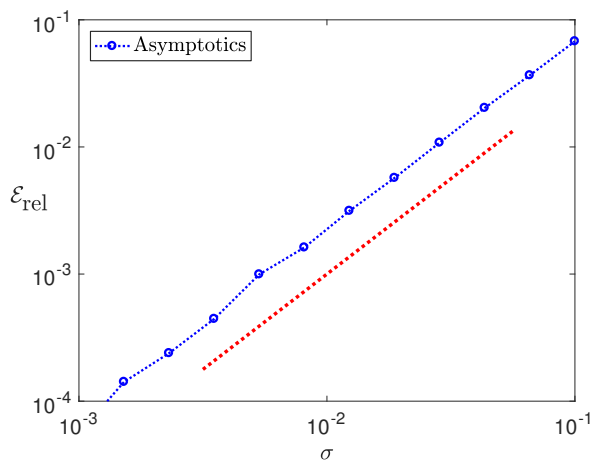

(b) rescaled $\bar{\kappa} / \bar{\kappa}_{\text {bp }}$ against $\sqrt{\sigma}$

FIG. 9. Heterogeneous lattice example. Comparison of the asymptotic formula (5.32) with KMC simulations. Left: plot of $\bar{\kappa} / \bar{\kappa}_{b p}$ against $\sqrt{\sigma}$. Right: relative error against $\sigma$. Line (red dotted) of slope $3 / 2$ added for comparison.

6.1.3. Example: Heterogeneous lattice. For this example, we consider a repeating cell $\mathcal{P}=\left[-\frac{1}{2}, \frac{1}{2}\right]^{2}$ with four pores centered at

$$
\mathbf{x}_{1}=\left(\frac{1}{4}, \frac{1}{4}\right), \quad \mathbf{x}_{2}=\left(-\frac{1}{4}, \frac{1}{4}\right), \quad \mathbf{x}_{3}=\left(-\frac{1}{4},-\frac{1}{4}\right), \quad \mathbf{x}_{4}=\left(\frac{1}{4},-\frac{1}{4}\right) .
$$

The pores are circular with radii $\varepsilon$ or $\varepsilon / 4$ so that the capacitance vector is $\mathbf{c}=(2 \varepsilon / \pi) \mathbf{a}$, where $\mathbf{a}=\left[\frac{1}{4}, 1, \frac{1}{4}, 1\right]$. We evaluate the Green's function matrix for this example to be

$$
\mathcal{G}=\left[\begin{array}{llll}
0.6207 & 0.1818 & 0.1818 & 0.2571 \\
0.1818 & 0.6207 & 0.2571 & 0.1818 \\
0.1818 & 0.2571 & 0.6207 & 0.1818 \\
0.2571 & 0.1818 & 0.1818 & 0.6207
\end{array}\right]
$$

and form an approximation for $\bar{\kappa} / \bar{\kappa}_{\text {bp }}$ from (5.32). The convergence results are shown in Figure 9. In the particular case with nonuniform pore sizes, the corresponding BP leakage parameter is

$$
\bar{\kappa}_{\mathrm{bp}}=\frac{4 \sigma}{\pi \varepsilon} \frac{N \bar{a}}{\|\mathbf{a}\|^{2}}=\frac{4 \sigma}{\pi \varepsilon}\left(\frac{\sum_{j=1}^{N} a_{j}}{\sum_{j=1}^{N} a_{j}^{2}}\right) .
$$

6.2. Optimizing capture rates. Our asymptotics allow us to extend some of the observations made by previous researchers on which configurations optimize capture rates for the diffuse pore asymptotics. In the homogenized version of the problem, the capture rate increases monotonically with the leakage parameter $\bar{\kappa}$. As such, we use our asymptotic results to seek configurations that maximize $\bar{\kappa}$.

At the outset, we note that for a given configuration of pores, at leading order

$$
\bar{\kappa}=\frac{2 \pi \varepsilon N \bar{c}}{|\mathcal{P}|}=\frac{2 \pi \varepsilon}{|\mathcal{P}|}\left(\sum_{k=1}^{N} c_{k}\right),
$$

from which we can see that increasing the capacitances of the individual pores increases $\bar{\kappa}$. Moreover, increasing the size of the periodic cell, $\mathcal{P}$, will decrease the capture rate. A natural optimization problem can also be considered: Among all regular 
lattices of identical pores with fixed area fraction $\sigma$, which one maximizes the capture rate? We can answer this question in the dilute pore limit via our asymptotics. As the pores have equal capacitances (here $\varepsilon \bar{c}$ ), the third term of the asymptotic expansion for $\bar{\kappa}^{-1}$ vanishes and it is convenient to consider (5.32), which reduces to

$$
\bar{\kappa} \sim \frac{2 \pi \varepsilon \bar{c}}{|\mathcal{P}|}[1-2 \pi \varepsilon \bar{c} \bar{R}(\mathbf{p}, \mathbf{q})]^{-1} .
$$

We wish to maximize $\bar{\kappa}$, which is equivalent to maximizing $\bar{R}(\mathbf{p}, \mathbf{q})$, while $|\mathcal{P}|=|\mathbf{p} \times \mathbf{q}|$ is fixed to unity.

6.2.1. Example: Rectangular lattices. In this example, we restrict ourselves to homogeneous rectangular lattices with primitive cell $\mathcal{P}=\left[-\frac{a}{2}, \frac{a}{2}\right] \times\left[\frac{b}{2}, \frac{b}{2}\right]$ and $|\mathcal{P}|=a b=1$. This yields

$$
\bar{\kappa} \sim 2 \pi \varepsilon \bar{c}[1-2 \pi \varepsilon \bar{c} \bar{R}]^{-1} .
$$

In Figure 10, we observe that the optimal lattice is a square $(a / b=1)$. However, in the space of general two-dimensional Bravais lattices, we find (cf. section 6.2.2) the square lattice to be a saddle point. For a sufficiently elongated rectangular cell $a / b \geq 7.055$, the regular part $\bar{R}$ becomes negative. This indicates that interpore competition enhances $\bar{\kappa}$ for a nearly square domain (and deprecates it for sufficiently elongated domains) relative to the $\mathrm{BP}$ value (cf. 6.3).

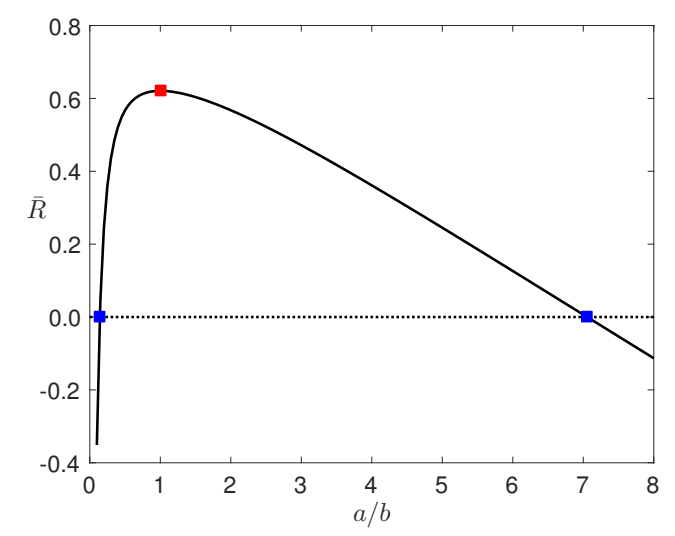

FIG. 10. The regular part $\bar{R}$ for rectangular lattices of aspect ratio $a / b$. A square lattice (red square) maximizes $\bar{R}$ and hence $\bar{\kappa}$, while $\bar{R}$ becomes negative for narrow rectangular lattices. Color is available online only.

6.2.2. Example: General Bravais lattices. There is a long history of minimizing various interaction energies over the space of two-dimensional Bravais lattices [31, 40] that continues to be an active area of research at present [13, 14, 15]. These studies concentrate on lattice sums, which have the same symmetries as the regular part of the Green's function, $\bar{R}$, which we are studying here. It is striking that many of these studies find that the hexagonal lattice is a global extremum, as we will demonstrate numerically here.

Our strategy for numerical investigation of the capture rate of general twodimensional lattices is to first parameterize the space of possible lattices and then 
rescale them to correspond to a primitive cell of area unity. By rotating and rescaling, we can reduce the space of lattices to a two-parameter family, $(p, q)$, given by

$$
\mathbf{p}=\left(\frac{p}{\sqrt{q}}, \sqrt{q}\right), \quad \mathbf{q}=\left(\frac{1}{\sqrt{q}}, 0\right)
$$

where $q>0$. However, this set of vectors has an additional set of symmetries generated by translations $(\mathbf{p}, \mathbf{q}) \rightarrow(\mathbf{p}+\mathbf{q}, \mathbf{q})$ which is equivalent to $(p, q) \rightarrow(p+1, q)$, exchanging the two vectors $(\mathbf{p}, \mathbf{q}) \rightarrow(\mathbf{q}, \mathbf{p})$, which is equivalent to the inversion $(p, q) \rightarrow$ $\left(p, \frac{q}{\sqrt{p^{2}+q^{2}}}\right)$ and the reflection $(\mathbf{p}, \mathbf{q}) \rightarrow(-\mathbf{p}, \mathbf{q})$ equivalent to $(p, q) \rightarrow(-p, q)$. The reflection and translation symmetries can also be combined to yield a mirror symmetry $(p, q) \rightarrow(1-p, q)$. These correspond to the symmetries of a Poincaré half-plane, and every configuration occurs infinitely often in a fractal pattern well known in the study of hyperbolic geometry. All configurations occur in the basic cell $\frac{1}{2} \leq p \leq 1$, $q \geq \sqrt{1-p^{2}}$.

In the contour plot of $\bar{R}(\mathbf{p}, \mathbf{q})$ displayed in Figure 11, we observe two types of critical points: the hexagonal lattice corresponding to the global maxima of $\bar{R}(\mathbf{p}, \mathbf{q})$, which occurs at $(p, q)=\left(\frac{1}{2}, \frac{\sqrt{3}}{2}\right)$ in the basic cell; and the square lattice, which is a saddle point which occurs at $(p, q)=(1,1)$ in the basic cell. There is an infinite sequence of images of these critical points, arising from the symmetries and manifested by a fractal set in the contour plot. Our conclusion is that hexagonal lattices maximize the rate of absorption.

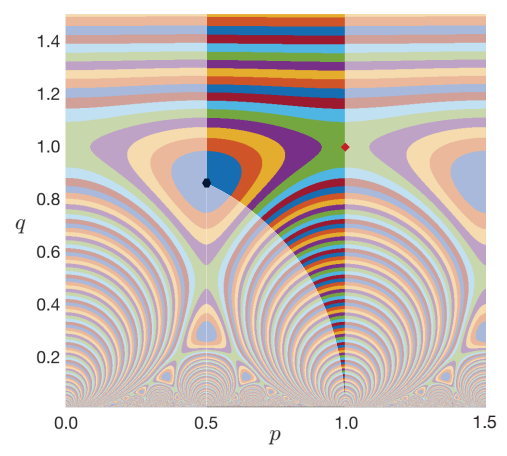

FIG. 11. Contour plot of $\bar{R}(\mathbf{p}, \mathbf{q})$ over two-dimensional lattices where $(p, q)$ are described in (6.7). The function $\bar{R}$ has the symmetries of the Poincaré half-plane; the values in the basic cell (the bright region $1 / 2<p<1$ and $p^{2}+q^{2}>1$ ) are repeated infinitely often under the reflection and inversion symmetries. In the basic cell, there is a maximum (navy hexagon) at $(p, q)=\left(\frac{1}{2}, \frac{\sqrt{3}}{2}\right)$ corresponding to the hexagonal lattice and a saddle point (red square) which occurs at $(p, q)=(1,1)$ corresponding to the square lattice. Each of these critical points has an infinite number of images which accumulate on the line $q=0$.

7. Discussion. This work has considered the capture rate of diffusing molecules on an infinite plane with a periodic array of absorbing surface pores. Our analytical treatment is based on boundary homogenization, an approach which seeks to replace the complex configuration of Neumann and Dirichlet surface conditions with a single Robin condition that matches the expected lifetime of the diffusing particle.

Previous work on this longstanding problem has focused on square lattices of homogeneous (common capacitance) pores. The first contribution of this paper is 
to obtain boundary homogenizations for heterogeneous configurations of pores on general Bravais lattices. These results are derived in the limit of a small absorbing area fraction and rely on detailed knowledge of the periodic Green's function for the Laplacian. We introduce a lattice sum for this quantity and discuss techniques for its rapid evaluation on general Bravais lattices. This significantly improves on discretization-based approximations used previously [16]. We find that a hexagonal lattice maximizes the absorption rate on the surface.

As a confirmation of our theoretical results, we introduced a novel kinetic Monte Carlo (KMC) method for efficient and accurate sampling of the capture time distribution described by (1.1). This method is based on a two-stage decomposition of Brownian paths into those transiting from bulk to plane (Stage I) and plane to bulk (Stage II). In each of these stages, the terminus and duration of the sojourn are sampled from exact distributions. This approach improves on previous methods that use spherical projectors [4], as it eliminates arbitrary parameters which are needed to ensure convergence. Comparison to fitted approximations for $\bar{\kappa}$ shows the accuracy of the method and validates the new asymptotic results.

There are several avenues for future investigation arising from this work. It is desirable to extend KMC methods to general polyhedra which can represent curved surfaces. For closed surfaces in three dimensions, it is desirable to develop KMC methods that can accurately and efficiently simulate the Brownian paths that elude the target surface and escape to infinity. In biological applications, pores may be growing or shrinking and the surface itself may be in motion. A challenging avenue for future work would be to extend the KMC framework for evolving surfaces and dynamic pore geometries.

Appendix A. The periodic Green's function. The dilute fraction limit approximation of $\bar{\kappa}(5.32)$ is developed in terms of the periodic Green's function for the Laplacian. In this appendix, we solve for this function, which gives the response to a periodic set of sources on the bounding plane. Denote the open half-space of $\mathbf{x}=(\mathbf{y}, z) \in \mathbb{R}^{3}$, where $\mathbf{y}=(x, y) \in \mathbb{R}^{2}$ and $z>0$ as $\Omega$, and let $\partial \Omega$ be the bounding plane where $z=0$. The free space Green's function, $g(\mathbf{y}, z)$, for the Neumann problem on the half-space is

$$
g(\mathbf{y}, z) \equiv-\frac{1}{2 \pi} \frac{1}{|\mathbf{x}|}=-\frac{1}{2 \pi} \frac{1}{\sqrt{r^{2}+z^{2}}}
$$

where $r=|\mathbf{y}|=\sqrt{x^{2}+y^{2}}$. Let $\mathbf{p}$ and $\mathbf{q}$ be linearly independent vectors in $\mathbb{R}^{2}$, and consider the lattice

$$
\Lambda=\left\{m \mathbf{p}+n \mathbf{q} \mid(m, n) \in \mathbb{Z}^{2}\right\}
$$

on the plane. We can define a primitive cell, $\mathcal{P}$, which is the parallelogram

$$
\mathcal{P}=\left\{\alpha \mathbf{p}+\beta \mathbf{q}|| \alpha\left|<\frac{1}{2},\right| \beta \mid<\frac{1}{2}\right\}, \quad|\mathcal{P}|=|\mathbf{p} \times \mathbf{q}| .
$$

The plane can be partitioned into translates of this primitive cell (each centered on a lattice point in $\Lambda$ ) and the boundaries between them. We look for a solution where there is a unit flux source at each point of $\Lambda$ and which is periodic under translation by the vectors $\mathbf{p}$ and $\mathbf{q}$.

As a starting point, we might consider the periodic Neumann problem with sources 
at each point on $\Lambda$,

$$
\begin{gathered}
\Delta G(\mathbf{y}, z)=0 \quad \text { for }(\mathbf{y}, z) \in \Omega, \\
G_{z}(\mathbf{y}, 0)=\sum_{(m, n) \in \mathbb{Z}^{2}} \delta(\mathbf{y}-m \mathbf{p}-n \mathbf{q}), \quad \mathbf{y} \in \partial \Omega, \\
G(\mathbf{y}+m \mathbf{p}+n \mathbf{q}, z)=G(\mathbf{y}, z) \quad \text { for }(m, n) \in \mathbb{Z}^{2},(\mathbf{y}, z) \in \Omega,
\end{gathered}
$$

which seems solvable via superposition. This strategy faces several obstacles. First, the naive lattice sum solution one might propose,

$$
\sum_{(m, n) \in \mathbb{Z}^{2}} g(\mathbf{y}-m \mathbf{p}-n \mathbf{q}, z)=\sum_{(m, n) \in \mathbb{Z}^{2}}-\frac{1}{2 \pi} \frac{1}{\sqrt{|\mathbf{y}-m \mathbf{p}-n \mathbf{q}|^{2}+z^{2}}}
$$

does not converge. Second, the vertical flux through each cell of the lattice is finite, which implies a linear growth of $G$ as $z$ increases. Finally, the solution is arbitrary up to an additive constant. These problems were addressed for the case of a square lattice in [5], and we extend that analysis to a general lattice here.

Let us examine the vertical flux in a bit more detail. Consider the cylinder $\{(\mathbf{y}, z) \mid \mathbf{y} \in \mathcal{P}, 0<z<h\}$. The flux of $G$ through the lateral edges of this cylinder must be zero by the horizontal periodicity. The vertical flux through the bottom boundary is unity by the boundary condition (A.2b). As such, the total flux through the top of the cylinder at $z=h$ must also be unity:

$$
\iint_{\mathcal{P}} G_{z}(\mathbf{y}, h) d \mathbf{y}=1, \quad h>0 .
$$

We will argue below (again following the ideas in [5]) that as the height tends to infinity, horizontal variation in the solution decays exponentially and

$$
G(\mathbf{y}, z) \sim \frac{z}{|\mathcal{P}|}+G_{\infty}+\mathcal{O}(1) \quad \text { as } \quad z \rightarrow \infty
$$

Here, the coefficient of the linear term guarantees a unit vertical flux through each horizontal cross-section of the cylinder above $\mathcal{P}$. The solution for $G$ is arbitrary up to an additive constant, $G_{\infty}$, and we choose $G_{\infty}=0$ for simplicity.

Using this knowledge, we reformulate the problem to look for a doubly periodic solution with specified growth at large $z$ :

$$
\begin{gathered}
\Delta G(\mathbf{y}, z)=0 \quad \text { for }(\mathbf{y}, z) \in \Omega, \\
G_{z}(\mathbf{y}, 0)=\sum_{(m, n) \in \mathbb{Z}^{2}} \delta(\mathbf{y}-m \mathbf{p}-n \mathbf{q}), \quad \mathbf{y} \in \partial \Omega, \\
G(\mathbf{y}, z) \sim \frac{z}{|\mathcal{P}|}+\mathcal{O}(1) \quad \text { as } z \rightarrow \infty, \\
G(\mathbf{y}+m \mathbf{p}+n \mathbf{q}, z)=G(\mathbf{y}, z) \quad \text { for }(m, n) \in \mathbb{Z}^{2}, \quad(\mathbf{y}, z) \in \Omega .
\end{gathered}
$$

We now break the Green's function into two terms: a singular piece identical to the half-space Green's function, $g(\mathbf{y}, z)$, and a regular piece, $r(\mathbf{y}, z)$, that captures the horizontal variation, ensures periodicity, and satisfies the boundary condition at large $z$. Specifically, we have

$$
G(\mathbf{y}, z)=g(\mathbf{y}, z)+r(\mathbf{y}, z)
$$


We further break the regular portion into two pieces,

$$
r(\mathbf{y}, z)=\bar{R}+R(\mathbf{y}, z),
$$

where $\bar{R}=r(\mathbf{0}, 0)$ is a constant, specifically the value of the regular part of the Green's function at the origin, which implies that $R(\mathbf{0}, 0)=0$.

Following the ideas in [5], we propose that $R(\mathbf{y}, z)$ be given by

$$
\begin{aligned}
& R(\mathbf{y}, z)=\sum_{(m, n) \in \mathbb{Z}_{*}^{2}} g(\mathbf{y}-m \mathbf{p}-n \mathbf{q}, z)-g(-m \mathbf{p}-n \mathbf{q}, 0)-\mathbf{x} \cdot \nabla g(-m \mathbf{p}-n \mathbf{q}, 0) \\
& \left(\text { A.6) }=-\frac{1}{2 \pi} \sum_{(m, n) \in \mathbb{Z}_{*}^{2}}\left[\frac{1}{\sqrt{|\mathbf{y}-m \mathbf{p}-n \mathbf{q}|^{2}+z^{2}}}-\frac{1}{|m \mathbf{p}+n \mathbf{q}|}-\frac{(m \mathbf{p}+n \mathbf{q}) \cdot \mathbf{y}}{|m \mathbf{p}+n \mathbf{q}|^{3}}\right],\right.
\end{aligned}
$$

where $\mathbb{Z}_{*}^{2}$ is the set of ordered pairs of integers $(m, n)$ excluding $(0,0)$. To understand this expression, note that the first term is a lattice sum of translates of the halfspace Green's function. From each term in this sum, we have subtracted its linear power series approximation at the origin. The linear correction is harmonic, as are the translates of the Greens function, so the proposed solution satisfies (A.3a). The linear correction also ensures uniform convergence of the series. To see this, note that for fixed $\mathbf{y}$ and $z$,

$$
\left|\frac{1}{\sqrt{|\mathbf{y}-m \mathbf{p}-n \mathbf{q}|^{2}+z^{2}}}-\frac{1}{|m \mathbf{p}+n \mathbf{q}|}-\frac{(m \mathbf{p}+n \mathbf{q}) \cdot \mathbf{y}}{|m \mathbf{p}+n \mathbf{q}|^{3}}\right| \leq \frac{C}{\left(m^{2}+n^{2}\right)^{3 / 2}}
$$

for some $C$ (which may depend on $\mathbf{y}$ and $z$ ). Consequently, this sum is absolutely convergent. Finally, subtracting the linear correction ensures that both $R(\mathbf{0}, 0)$ and $\nabla R(\mathbf{0}, 0)$ vanish.

The proposed form of the periodic Green's function (A.4) satisfies the flux boundary condition (A.3b) at $z=0$. We must check that the Green's function is doubly periodic, (A.3d), and choose $\bar{R}$ to satisfy the boundary condition (A.3c) at large $z$.

A.1. Symmetries and periodicity. From the symmetries of (A.3a), $G(\mathbf{y}, z)$ is doubly periodic and even with respect to the lattice $\Lambda$. That is,

$$
\begin{aligned}
& G(\mathbf{y}+m \mathbf{p}+n \mathbf{q}, z)=G(\mathbf{y}, z) \quad \text { for } \quad(m, n) \in \mathbb{Z}^{2},(\mathbf{y}, z) \in \Omega \\
& G(\alpha \mathbf{p}+\beta \mathbf{q}, z)=G(-\alpha \mathbf{p}-\beta \mathbf{q}, z) \quad \text { for } \quad(\alpha, \beta) \in \mathbb{R}^{2}, z>0 .
\end{aligned}
$$

As $g(\mathbf{y}, z)$ is axisymmetric, (A.7b) implies that the regular part of the Green's function also has even symmetry at the origin with respect to the lattice:

$$
r(\alpha \mathbf{p}+\beta \mathbf{q}, z)=r(-\alpha \mathbf{p}-\beta \mathbf{q}, z) \quad \text { for } \quad(\alpha, \beta) \in \mathbb{R}^{2}, z>0 .
$$

Differentiating (A.8) with respect to $\alpha$ and $\beta$ and sending these parameters to zero implies that

$$
\mathbf{p} \cdot \nabla r(\mathbf{0}, z)=0, \quad \mathbf{q} \cdot \nabla r(\mathbf{0}, z)=0,
$$

which demonstrates that the horizontal component of the gradient vanishes when $\mathbf{y}=$ 0. Moreover, the flux boundary condition at $z=0,(\mathrm{~A} .3 \mathrm{~b})$, implies that $r_{z}(\mathbf{y}, 0)=0$, from which we deduce that the gradient of the regular part vanishes at the origin:

$$
\nabla r(\mathbf{0}, 0)=\mathbf{0} .
$$


We now show that our proposed solution (A.4), (A.6) is periodic under translation by any member of the lattice $\Lambda$. To prove this, we again use a modification of an argument in [5]. Consider the Hessian of our proposed solution for $G$ :

$$
\mathcal{H}_{i j}=\frac{\partial}{\partial x_{i}}\left(\frac{\partial G}{\partial x_{j}}\right)=\sum_{(m, n) \in \mathbb{Z}^{2}} \frac{\partial^{2} g}{\partial x_{i} \partial x_{j}}(\mathbf{y}-m \mathbf{p}-n \mathbf{q}, z) .
$$

This sum is absolutely convergent, doubly periodic by construction, and infinitely differentiable in $z>0$. Moreover, at $z=0$ the singular part of the Hessian matches the Hessian of the free space Green's function at each point in $\Lambda$ guaranteeing the proper singular behavior.

We wish now to reconstruct first the gradient of $G$ and then $G$ itself. While this may seem straightforward, there are several difficulties. First, integration may yield a nonperiodic function; Fourier analysis can be used to show that if the gradient of $G$ has nonzero mean value in the horizontal directions, then $G$ will have an associated linear growth (to illustrate this, consider the linear function $F=c_{1} x+c_{2} y$, which is not periodic and which has a gradient that is a constant vector which is periodic). Second, we must be careful to obtain a convergent (ideally absolutely convergent) series.

To reconstruct $G$, we first note that on a simply connected domain when two functions have the same gradient, they differ by at most a constant. We will now reconstruct the gradient from the Hessian (which is essentially the gradient of the gradient of $G$ ) and show that it is indeed doubly periodic.

Consider a line integral of the Hessian along a line, $\Gamma$, from the point $(\overline{\mathbf{y}}, \bar{z})$ to a point translated by one of the lattice vectors, say $(\overline{\mathbf{y}}+\mathbf{p}, \bar{z})$ :

$$
\begin{aligned}
\int_{\Gamma} \mathcal{H}_{i j} \cdot d \mathbf{x}_{i} & =\int_{\Gamma} \frac{\partial}{\partial x_{i}}\left(\frac{\partial G}{\partial x_{j}}\right) \cdot d \mathbf{x}_{i} \\
& =\frac{\partial G}{\partial x_{j}}(\overline{\mathbf{y}}+\mathbf{p}, \bar{z})-\frac{\partial G}{\partial x_{j}}(\overline{\mathbf{y}}, \bar{z}) \\
& =\sum_{(m, n) \in \mathbb{Z}^{2}} \frac{\partial g}{\partial x_{j}}(\overline{\mathbf{y}}+\mathbf{p}-m \mathbf{p}-n \mathbf{q}, \bar{z})-\frac{\partial g}{\partial x_{j}} g(\overline{\mathbf{y}}-m \mathbf{p}-n \mathbf{q}, \bar{z}) \\
& =\sum_{n=-\infty}^{\infty}\left[\sum_{m=-\infty}^{\infty} \frac{\partial g}{\partial x_{j}}(\overline{\mathbf{y}}-(m-1) \mathbf{p}-n \mathbf{q}, \bar{z})-\sum_{m=-\infty}^{\infty} \frac{\partial g}{\partial x_{j}}(\overline{\mathbf{y}}-m \mathbf{p}-n \mathbf{q}, \bar{z})\right] \\
& =0
\end{aligned}
$$

which guarantees that the integral of $\mathcal{H}_{i j}$ retains the doubly periodic symmetry of the lattice. Showing that the sum in this calculation vanishes is a bit subtle; we need to check that we have broken the inner sum into two absolutely convergent series. Note that

$$
\left|\frac{\partial g}{\partial x_{j}}(\overline{\mathbf{y}}-m \mathbf{p}-n \mathbf{q}, \bar{z})\right| \leq \frac{C_{1}+C_{2} m}{\left(m^{2}+n^{2}\right)^{3 / 2}}
$$

for some $C_{1}$ and $C_{2}$ (which may depend on $\overline{\mathbf{y}}, \bar{z}$, and $n$ ). Consequently, the inner sums in $m$ are absolutely convergent and one can show that their difference vanishes by observing that they are re-indexed versions of the same sum.

We now wish to construct the gradient of the periodic Green's function explicitly. We do so in a fashion that will make the gradient of the regular portion of the Green's function vanish at the origin (A.9). Let $\gamma$ be a path from the origin to a point $(\mathbf{y}, z)$, and write the gradient as the sum of the gradients of the singular and regular portions 
of the periodic Green's function:

$$
\frac{\partial G}{\partial x_{j}}=\frac{\partial g}{\partial x_{j}}(\mathbf{y}, z)+\frac{\partial r}{\partial x_{j}}(\mathbf{y}, z)
$$

We now identify

$$
\begin{aligned}
\frac{\partial r}{\partial x_{j}}(\mathbf{y}, z) & =\sum_{(m, n) \in \mathbb{Z}_{*}^{2}} \int_{\gamma} \frac{\partial^{2} g}{\partial x_{i} \partial x_{j}}(\mathbf{y}-m \mathbf{p}-n \mathbf{q}, z) \cdot d \mathbf{x}_{i} \\
& =\sum_{(m, n) \in \mathbb{Z}_{*}^{2}} \frac{\partial g}{\partial x_{j}}(\mathbf{y}-m \mathbf{p}-n \mathbf{q}, z)-\frac{\partial g}{\partial x_{j}}(-m \mathbf{p}-n \mathbf{q}, 0) .
\end{aligned}
$$

This yields an absolutely convergent sum for the regular part of the Green's function which vanishes at the origin. This expression for the gradient of the Green's function is guaranteed to be periodic by the calculation above. By construction (or direct substitution), the gradient of the regular part vanishes at the origin, $\frac{\partial r}{\partial x_{j}}(\mathbf{0}, 0)=0$. Moreover, the gradient of the periodic Green's function acquires the symmetry of the free space Green's function; it is odd in the horizontal component $(\sigma=1)$ and even in the vertical component $(\sigma=0)$ with respect to translation on the lattice:

$$
\frac{\partial G}{\partial x_{j}}(\alpha \mathbf{p}+\beta \mathbf{q}, z)=(-1)^{\sigma} \frac{\partial G}{\partial x_{j}}(-\alpha \mathbf{p}-\beta \mathbf{q}, z) \quad \text { for }(\alpha, \beta) \in \mathbb{R}^{2}, z>0 .
$$

We now proceed to reconstruct $G$ from its gradient. One first needs to show that $G$ is periodic. We can use an argument similar to that used above. Once again consider an integral along a line segment, $\Gamma$, from the point $(\overline{\mathbf{y}}, \bar{z})$ to a point translated by one of the lattice vectors, say $(\overline{\mathbf{y}}+\mathbf{p}, \bar{z})$ :

$$
G(\overline{\mathbf{y}}+\mathbf{p}, \bar{z})-G(\overline{\mathbf{y}}, \bar{z})=\int_{\Gamma} \frac{\partial G}{\partial x_{i}}(\mathbf{x}, \bar{z}) \cdot d \mathbf{x}_{i}=0 .
$$

Here, we know the integral vanishes because it is taken over a full period of an odd periodic function. Consequently, $G(\mathbf{y}, z)$ is doubly periodic and satisfies the boundary condition (A.3d). We now construct the periodic Green's function explicitly. Recalling that $\bar{R}=r(\mathbf{0}, 0)$ is the value of the regular part of the periodic Green's function at the origin, we again integrate along a path $\gamma$ from the origin to a point $(\mathbf{y}, z)$,

$$
\begin{aligned}
R(\mathbf{y}, z) & =r(\mathbf{y}, z)-\bar{R}=r(\mathbf{y}, z)-r(\mathbf{0}, 0) \\
& =\int_{\gamma} \frac{\partial r}{\partial x_{j}}(\mathbf{y}, z) \cdot d \mathbf{x}_{i} \\
& =\sum_{(m, n) \in \mathbb{Z}_{*}^{2}} \int_{\gamma}\left[\frac{\partial g}{\partial x_{i}}(\mathbf{y}-m \mathbf{p}-n \mathbf{q}, z)-\frac{\partial g}{\partial x_{i}}(-m \mathbf{p}-n \mathbf{q}, 0)\right] \cdot d \mathbf{x}_{i} \\
& =\sum_{(m, n) \in \mathbb{Z}_{*}^{2}} g(\mathbf{y}-m \mathbf{p}-n \mathbf{q}, z)-g(-m \mathbf{p}-n \mathbf{q}, 0)-\mathbf{x} \cdot \nabla g(-m \mathbf{p}-n \mathbf{q}, 0) \\
& =-\frac{1}{2 \pi} \sum_{(m, n) \in \mathbb{Z}_{*}^{2}}\left[\frac{1}{\sqrt{|\mathbf{y}-m \mathbf{p}-n \mathbf{q}|^{2}+z^{2}}}-\frac{1}{|m \mathbf{p}+n \mathbf{q}|}-\frac{(m \mathbf{p}+n \mathbf{q}) \cdot \mathbf{y}}{|m \mathbf{p}+n \mathbf{q}|^{3}}\right],
\end{aligned}
$$

as previously advertised. Note that we still need to determine $\bar{R}$ to ensure the large $z$ boundary condition (A.3c) is satisfied. 
A.2. Large $z$ behavior. We can use asymptotics and Riemann sums to evaluate the behavior of $R(y, z)$ as $z \rightarrow \infty$. Let $\Delta=1 / z$, and define $\xi_{m}=m \Delta$ and $\eta_{n}=n \Delta$. We can rewrite (A.6) as

$$
\begin{aligned}
R(\mathbf{y}, z) & =-\frac{1}{2 \pi} \sum_{(m, n) \in \mathbb{Z}_{*}^{2}}\left[\frac{1}{\sqrt{|\mathbf{y}-m \mathbf{p}-n \mathbf{q}|^{2}+z^{2}}}-\frac{1}{|m \mathbf{p}+n \mathbf{q}|}-\frac{(m \mathbf{p}+n \mathbf{q}) \cdot \mathbf{y}}{|m \mathbf{p}+n \mathbf{q}|^{3}}\right] \\
& =-\frac{z}{2 \pi} \sum_{(m, n) \in \mathbb{Z}_{*}^{2}}\left[\frac{\Delta^{2}}{\sqrt{1+\left|\Delta \mathbf{y}-\xi_{m} \mathbf{p}-\eta_{n} \mathbf{q}\right|^{2}}}-\frac{\Delta^{2}}{\left|\xi_{m} \mathbf{p}+\eta_{n} \mathbf{q}\right|}-\frac{\Delta^{3}\left(\xi_{m} \mathbf{p}+\eta_{n} \mathbf{q}\right) \cdot \mathbf{y}}{\left|\xi_{m} \mathbf{p}+\eta_{n} \mathbf{q}\right|^{3}}\right] \\
& \sim-\frac{z}{2 \pi} \sum_{(m, n) \in \mathbb{Z}_{*}^{2}}\left[\frac{\Delta^{2}}{\sqrt{1+\left|\xi_{m} \mathbf{p}+\eta_{n} \mathbf{q}\right|^{2}}}-\frac{\Delta^{2}}{\left|\xi_{m} \mathbf{p}+\eta_{n} \mathbf{q}\right|}\right]+\mathcal{O}(1) .
\end{aligned}
$$

Here, we have expanded as $\Delta \rightarrow 0$ while holding $\xi_{m}$ and $\eta_{n}$ constant. The $\mathcal{O}\left(\Delta^{3}\right)$ terms in the summand cancel, and the $\mathcal{O}\left(\Delta^{4}\right)$ terms eventually yield a summation that contributes a term of order $1 / z$ to $R(\mathbf{y}, z)$.

The summation is a Riemann sum for a singular integral in two dimensions. Define

$$
S(\Delta)=-\frac{1}{2 \pi} \sum_{(m, n) \in \mathbb{Z}_{*}^{2}}\left[\frac{\Delta^{2}}{\sqrt{1+\left|\xi_{m} \mathbf{p}+\eta_{n} \mathbf{q}\right|^{2}}}-\frac{\Delta^{2}}{\left|\xi_{m} \mathbf{p}+\eta_{n} \mathbf{q}\right|}\right], \quad \xi_{m}=m \Delta, \quad \eta_{n}=n \Delta,
$$

and consider the behavior as $\Delta$ tends to zero. This is a Riemann sum for an integral but with an integrable singularity at the origin. Standard methods ensure that the Riemann sum for this integrable singularity converges, but the error term is $\mathcal{O}(\Delta)$ (as opposed to the normal $\mathcal{O}\left(\Delta^{2}\right)$ ) due to the contributions from the singularity at the origin:

$$
\lim _{\Delta \rightarrow 0} S(\Delta)=-\frac{1}{2 \pi} \int_{\xi=-\infty}^{\infty} \int_{\eta=-\infty}^{\infty}\left[\frac{1}{\sqrt{1+|\xi \mathbf{p}+\eta \mathbf{q}|^{2}}}-\frac{1}{|\xi \mathbf{p}+\eta \mathbf{q}|}\right] d \xi d \eta+R_{\infty} \Delta+\mathcal{O}\left(\Delta^{2}\right) .
$$

To evaluate the integral, first let $\mathbf{y}=\xi \mathbf{p}+\eta \mathbf{q}$ and then note that the resulting integral has polar symmetry. Letting $r=|\mathbf{y}|$, the area element transforms as

$$
d \xi d \eta=\frac{1}{|\mathcal{P}|} d \mathbf{y}=\frac{1}{|\mathcal{P}|} 2 \pi r d r
$$

which yields

$$
\begin{aligned}
& -\frac{1}{2 \pi} \int_{\xi=-\infty}^{\infty} \int_{\eta=-\infty}^{\infty}\left[\frac{1}{\sqrt{1+|\xi \mathbf{p}+\eta \mathbf{q}|^{2}}}-\frac{1}{|\xi \mathbf{p}+\eta \mathbf{q}|}\right] d \xi d \eta \\
= & -\frac{1}{|\mathcal{P}|} \int_{r=0}^{\infty}\left[\frac{1}{\sqrt{1+r^{2}}}-\frac{1}{r}\right] r d r=\frac{1}{|\mathcal{P}|} .
\end{aligned}
$$

This yield the large $z$ behavior as

$$
R(\mathbf{y}, z) \sim \frac{z}{|\mathcal{P}|}+R_{\infty}+\mathcal{O}(1)
$$

and we can identify that $R_{\infty}=-\bar{R}$. This expression can be used to evaluate $\bar{R}$ numerically, and while we have used this as a check on our calculations, a much more efficient method for computing $\bar{R}$ is described below. Crucially, we have verified that the solution (A.6) satisfies the far-field boundary condition (A.3c). 
A.3. A separation of variables solutions and exponential decay. We can find a separation of variables solution for the Green's function that satisfies (A.3). Define the reciprocal lattice vectors

$$
\mathbf{k}=2 \pi \frac{\hat{\mathbf{z}} \times \mathbf{p}}{|\mathcal{P}|}, \quad \mathbf{l}=2 \pi \frac{\hat{\mathbf{z}} \times \mathbf{q}}{|\mathcal{P}|},
$$

where $\hat{\mathbf{z}}$ is the vertical unit normal and (with mild abuse of notation) the operation $\hat{\mathbf{z}} \times \mathbf{v}$ rotates a vector $\mathbf{v}$ in the $(x, y)$ plane by $\pi / 2$ in a right-handed sense. This implies that

$$
(n \mathbf{k}+m \mathbf{l}) \cdot\left(m^{\prime} \mathbf{p}+n^{\prime} \mathbf{q}\right)=2 \pi\left(n n^{\prime}-m m^{\prime}\right)
$$

and guarantees the appropriate periodicity of the Green's function,

$$
\begin{aligned}
G(x, y, z) & =\frac{z}{|\mathcal{P}|}+\sum_{(m, n) \in \mathbb{Z}_{*}^{2}} a_{m n} e^{i(n \mathbf{k}+m \mathbf{l}) \cdot \mathbf{y}} e^{-|n \mathbf{k}+m \mathbf{l}| z}, \\
a_{m n} & =-\frac{1}{|\mathcal{P}||n \mathbf{k}+m \mathbf{l}|}=-\frac{1}{2 \pi} \frac{1}{|n \mathbf{p}+m \mathbf{q}|}
\end{aligned}
$$

which converges uniformly for $z>0$. At $z=0$, the flux converges in a distributional sense to $G_{z}(x, y, 0)=\delta(\mathbf{y})$. This demonstrates that the solution decays exponentially at large $z$ to the linear profile $G(x, y, z) \sim \frac{z}{|\mathcal{P}|}$.

A.4. Numerical evaluation of $\boldsymbol{R}(\mathrm{y}, 0)$. Our asymptotics require evaluating the lattice sum for $R(\mathbf{y}, 0)$ and the constant $\bar{R}$. There are a variety of effective strategies for doing this (such as Ewald summation [51]), but some experimentation has suggested that a combination of direct evaluation and Richardson extrapolation can rapidly yield high accuracy (up to one part in $10^{8}$ ) approximations. Define the partial sum

$$
\begin{aligned}
S(M) & =-\frac{1}{2 \pi} \sum_{\substack{m=-M \\
(m, n) \neq(0,0)}}^{M} \sum_{\substack{n=-M \\
\text { M }}}^{M} \frac{1}{\sqrt{|\mathbf{y}-m \mathbf{p}-n \mathbf{q}|^{2}}}-\frac{1}{|m \mathbf{p}+n \mathbf{q}|}-\frac{(m \mathbf{p}+n \mathbf{q}) \cdot \mathbf{y}}{|m \mathbf{p}+n \mathbf{q}|^{3}} \\
& =-\frac{1}{2 \pi} \sum_{\substack{m=-M \\
(m, n) \neq(0,0)}}^{M} \sum_{\substack{n=-M \\
\text { (m) }}}^{M} \frac{1}{\sqrt{|\mathbf{y}-m \mathbf{p}-n \mathbf{q}|^{2}}}-\frac{1}{|m \mathbf{p}+n \mathbf{q}|}
\end{aligned}
$$

Because the sum is finite, the term linear in $\mathbf{y}$ exactly cancels due to its odd symmetry in $m$ and $n$. For $M$ large, the error in the sum can be approximated as an integral (and corrections could be computed using an Euler-Maclaurin-type expansion). We merely note that the error has an asymptotic expansion:

$$
R(\mathbf{y}, 0)-S(M) \sim \frac{c_{1}}{M}+\frac{c_{2}}{M^{2}}+\cdots \quad \text { for } \quad M \gg 1 .
$$

Armed with this knowledge, we evaluate the sum for $M=2^{j}$, where $j$ is chosen to be a sequence of positive integers, and apply Richardson extrapolation. In practice, choosing $j=5,6,7,8,9$ yields accuracy to one part in $10^{8}$.

A.5. Numerical evaluation of $\overline{\boldsymbol{R}}$. We will use a lattice sum identity to evaluate $\bar{R}$. A past work [16] used numerical solutions of the PDE to estimate that $\bar{R} \approx 0.6207$ for a square lattice. Our method allows us to verify this result directly as an infinite sum in a few seconds of direct computation. 
For clarity, let us extend the notation for the Green's function to indicate explicitly the dependence on the lattice basis vectors $\mathbf{p}$ and $\mathbf{q}$ :

$$
G(\mathbf{y}, z ; \mathbf{p}, \mathbf{q})=g(\mathbf{y}, z)+\bar{R}(\mathbf{p}, \mathbf{q})+R(\mathbf{y}, z ; \mathbf{p}, \mathbf{q}) .
$$

A uniform linear rescaling of the spatial variables for this problem by a scale factor $\gamma$ yields the relationship

$$
G(\gamma \mathbf{y}, \gamma z ; \gamma \mathbf{p}, \gamma \mathbf{q})=\frac{1}{\gamma} G(\mathbf{y}, z ; \mathbf{p}, \mathbf{q}),
$$

and in fact this rescaling applies to all the individual terms also:

$$
\begin{aligned}
g(\gamma \mathbf{y}, \gamma z) & =\frac{1}{\gamma} g(\gamma \mathbf{y}, \gamma z), \\
\bar{R}(\gamma \mathbf{p}, \gamma \mathbf{q}) & =\frac{1}{\gamma} \bar{R}(\mathbf{p}, \mathbf{q}), \\
R(\gamma \mathbf{y}, \gamma z ; \gamma \mathbf{p}, \gamma \mathbf{q}) & =\frac{1}{\gamma} R(\mathbf{y}, z ; \mathbf{p}, \mathbf{q}) .
\end{aligned}
$$

To evaluate $\bar{R}$, we use the fact that a lattice $\Lambda$ can be written as the sum of four lattices of twice the period:

$$
\begin{aligned}
G(\mathbf{y}, z ; \mathbf{p}, \mathbf{q})= & G(\mathbf{y}, z ; 2 \mathbf{p}, 2 \mathbf{q})+G(\mathbf{y}+\mathbf{p}, z ; 2 \mathbf{p}, 2 \mathbf{q}) \\
& +G(\mathbf{y}+\mathbf{q}, z ; 2 \mathbf{p}, 2 \mathbf{q})+G(\mathbf{y}+\mathbf{p}+\mathbf{q}, z ; 2 \mathbf{p}, 2 \mathbf{q}) .
\end{aligned}
$$

We note that our choice of normalization means this sum exactly satisfies the large $z$ far-field condition (A.3c). The terms $G(\mathbf{y}, z ; \mathbf{p}, \mathbf{q})$ and $G(\mathbf{y}, z ; 2 \mathbf{p}, 2 \mathbf{q})$ are both singular at the origin, but if we subtract the free space Green's function $g(\mathbf{y}, z)$ from each side, the limit at the origin yields

$$
\begin{aligned}
\bar{R}(\mathbf{p}, \mathbf{q})= & g(\mathbf{p}, 0)+g(\mathbf{q}, 0)+g(\mathbf{p}+\mathbf{q}, 0)+R(\mathbf{p}, 0 ; 2 \mathbf{p}, 2 \mathbf{q}) \\
& +R(\mathbf{q}, 0 ; 2 \mathbf{p}, 2 \mathbf{q})+R(\mathbf{p}+\mathbf{q}, 0 ; 2 \mathbf{p}, 2 \mathbf{q})+4 \bar{R}(2 \mathbf{p}, 2 \mathbf{q})
\end{aligned}
$$

where we have used the fact that $R(\mathbf{0}, 0 ; \mathbf{p}, \mathbf{q})=0$ for any lattice. From the rescaling symmetry, we know that $\bar{R}(\mathbf{p}, \mathbf{q})=2 \bar{R}(2 \mathbf{p}, 2 \mathbf{q})$, which allows us to deduce that

$$
\begin{aligned}
\bar{R}(\mathbf{p}, \mathbf{q})= & -[g(\mathbf{p}, 0)+g(\mathbf{q}, 0)+g(\mathbf{p}+\mathbf{q}, 0)+R(\mathbf{p}, 0 ; 2 \mathbf{p}, 2 \mathbf{q}) \\
& +R(\mathbf{q}, 0 ; 2 \mathbf{p}, 2 \mathbf{q})+R(\mathbf{p}+\mathbf{q}, 0 ; 2 \mathbf{p}, 2 \mathbf{q})] .
\end{aligned}
$$

Consequently, we can compute $\bar{R}$ numerically from our algorithm for calculating $R(\mathbf{y}, 0)$ described above.

Acknowledgments. The authors acknowledge useful conversations with M. J. Ward and C. Muratov. The first author thanks the IMA at the University of Minnesota for hosting him in the Fall of 2017. Much of this work was prototyped as part of the third author's senior thesis at Harvey Mudd College, Claremont, CA, in 2017.

\section{REFERENCES}

[1] H. Ammari And H. Kang, Reconstruction of Small Inhomogeneities from Boundary Measurements, Springer, 2004.

[2] H. Ammari and H. Kang, Polarization and Moment Tensors, Springer, 2007. 
[3] R. W. BaKer And B. T. Low, Gas separation membrane materials: A perspective, Macromolecules, 47 (2014), pp. 6999-7013.

[4] L. Batsilas, A. M. Berezhrovskit, and S. Y. Shvartsman, Stochastic model of autocrine and paracrine signals in cell culture assays, Biophys. J., 85 (2003), pp. 3659-3665.

[5] A. G. Belyaev, G. A. Chechin, and R. R. Gadyl'shin, Effective membrane permeability: Estimates and low concentration asymptotics, SIAM J. Appl. Math., 60 (1999), pp. 84-108, https://doi.org/10.1137/S0036139996312880.

[6] A. M. Berezhrovskit, L. Dagdug, V. A. Lizunov, J. Zimmerberg, and S. M. Bezrukov, Communication: Clusters of absorbing disks on a reflecting wall: Competition for diffusing particles, J. Chem. Phys., 136 (2012), 211102.

[7] A. M. Berezhrovskit, L. Dagdug, V. A. Lizunov, J. Zimmerberg, and S. M. Bezrukov, Trapping by clusters of channels, receptors, and transporters: Quantitative description, Biophys. J., 106 (2014), pp. 500-509.

[8] A. M. Berezhkovskit, L. Dagdug, M.-V. Vazquez, V. A. Lizunov, J. Zimmerberg, and S. M. BezRukov, Trapping of diffusing particles by clusters of absorbing disks on a reflecting wall with disk centers on sites of a square lattice, J. Chem. Phys., 138 (2013), 064105.

[9] A. M. Berezhrovskit, Y. A. Makhnovskit, M. I. Monine, V. Y. Zitserman, and S. Y. Shvartsman, Boundary homogenization for trapping by patchy surfaces, J. Chem. Phys., 121 (2004), pp. 11390-11394.

[10] A. M. Berezhkovskit, M. I. Monine, C. B. Muratov, and S. Y. Shvartsman, Homogenization of boundary conditions for surfaces with regular arrays of traps, J. Chem. Phys., 124 (2006), 036103.

[11] H. C. Berg And E. M. Purcell, Physics of chemoreception, Biophys J., 20 (1977), pp. 193219.

[12] A. J. Bernoff And A. E. Lindsay, Numerical approximation of diffusive capture rates by planar and spherical surfaces with absorbing pores, SIAM J. Appl. Math., 78 (2018), pp. 266290, https://doi.org/10.1137/17M1124802.

[13] L. BÉTERmin, Local variational study of $2 D$ lattice energies and application to Lennard-Jones type interactions, Nonlinearity, 31 (2018), pp. 3973-4005.

[14] L. BÉtermin, Two-dimensional theta functions and crystallization among Bravais lattices, SIAM J. Math. Anal., 48 (2016), pp. 3236-3269, https://doi.org/10.1137/15M101614X.

[15] L. BÉTERmin AND P. Zhang, Minimization of energy per particle among Bravais lattices in $\mathbb{R}^{2}$ : Lennard-Jones and Thomas-Fermi cases, Commun. Contemp. Math., 17 (2015), 1450049.

[16] M. Bruna, S. J. Chapman, And G. Z. Ramon, The effective flux through a thin-film composite membrane, EPL (Europhysics Letters), 110 (2015), 40005.

[17] H. S. Carslaw and J. C. Jaeger, Heat in Solids, Vol. 1, Clarendon Press, 1959.

[18] A. Cheviakov and M. J. Ward, Optimizing the principal eigenvalue of the Laplacian in a sphere with interior traps, Math. Comput. Modelling, 53 (2011), pp. 1394-1409.

[19] F. C. Collins and G. E. Kimball, Diffusion-controlled reaction rates, J. Colloid Sci., 4 (1949), pp. $425-437$.

[20] R. Durrett, Probability: Theory and Examples, Cambridge University Press, 2010.

[21] A. S. HARDING AND J. F. HANCOCK, Using plasma membrane nanoclusters to build better signaling circuits, Trends Cell Biol., 18 (2008), pp. 364-371.

[22] D. Holcman and Z. Schuss, The narrow escape problem, SIAM Rev., 56 (2014), pp. 213-257, https://doi.org/10.1137/120898395.

[23] K. H. Keller AND T. R. Stein, A two-dimensional analysis of porous membrane transport, Math. Biosci., 1 (1967), pp. 421-437.

[24] T. Kolokolnikov, M. S. Titcombe, and M. J. Ward, Optimizing the fundamental Neumann eigenvalue for the Laplacian in a domain with small traps, European J. Appl. Math., 16 (2005), pp. 161-200.

[25] A. E. Lindsay, A. J. Bernoff, And M. J. Ward, First passage statistics for the capture of a Brownian particle by a structured spherical target with multiple surface traps, Multiscale Model. Simul., 15 (2017), pp. 74-109, https://doi.org/10.1137/16M1077659.

[26] A. E. Lindsay, T. Kolokolnikov, and J. C. Tzou, Narrow escape problem with a mixed trap and the effect of orientation, Phys. Rev. E (3), 91 (2015), 032111.

[27] S. Litwin, Monte Carlo simulation of particle adsorption rates at high cell concentration, Biophys. J., 31 (1980), pp. 271-277.

[28] V. A. Marchenko and E. Y. Khruslov, Homogenization of Partial Differential Equations, Birkhäuser, 2006.

[29] V. Maz'ya, A. Movchan, And M. Nieves, Green's Kernels and Meso-scale Approximations in Perforated Domains, Springer, 2013. 
[30] B. Y. Moizhes, Averaged electrostatic boundary conditions for metallic meshes, Zh. Tekh. Fiz., 25 (1955), pp. 167-176.

[31] H. L. Montgomery, Minimal theta functions, Glasg. Math. J., 30 (1988), pp. 75-85.

[32] C. B. Muratov and S. Y. Shvartsman, Boundary homogenization for periodic arrays of absorbers, Multicale Model. Simul., 7 (2008), pp. 44-61, https://doi.org/10.1137/070692832.

[33] S. H. NorThrup, Diffusion-controlled ligand binding to multiple competing cell-bound receptors, J. Phys. Chem., 92 (1988), pp. 5847-5850.

[34] T. Opplestrup, V. V. Bulatov, G. H. Gilmer, M. H. Kalos, and B. Sadigh, First-passage Monte Carlo algorithm: Diffusion without all the hops, Phys. Rev. Lett., 97 (2006), 230602.

[35] S. OzAwA, Singular variation of domains and eigenvalues of the Laplacian, Duke Math. J., 48 (1981), pp. $767-778$.

[36] S. V. Pageon, T. Tabarin, Y. Yamamoto, Y. Ma, P. R. Nicovich, J. S. Bridgeman, A. Cohnen, C. Benzing, Y. Gao, M. D. Crowther, K. Tungatt, G. Dolton, A. K. Sewell, D. A. Price, O. Acuto, R. G. Parton, J. J. Gooding, J. Rossy, J. Rossjohn, AND K. GAUS, Functional role of $t$-cell receptor nanoclusters in signal initiation and antigen discrimination, Proc. Natl. Acad. Sci. USA, 113 (2016), pp. E5454-E5463.

[37] G. Papanicolau, A. Bensoussan, and J.-L. Lions, Asymptotic Analysis for Periodic Structures, American Mathematical Society, 1978.

[38] G. A. Pavliotis And A. M. Stuart, Multiscale Methods: Averaging and Homogenization, Springer, 2008.

[39] S. Prager And H. L. Frisch, Interaction between penetration sites in diffusion through thin membranes, J. Chem. Phys., 62 (1975), pp. 89-91.

[40] R. A. Rankin, A minimum problem for the Epstein zeta-function, Glasg. Math. J., 1 (1953), pp. 149-158.

[41] J. Rossy, D. Williamson, C. Benzing, and K. Gaus, The integration of signaling and the spatial organization of the T cell synapse, Front. Immunol., 3 (2012), 352.

[42] S. Saddawi and W. Strieder, Size effects in reactive circular site interactions, J. Chem. Phys., 136 (2012), 044518.

[43] T. Saito, Nanocluster formation: More with memory, Immunity, 35 (2011), pp. 318-320.

[44] A. Saxena, B. P. Tripathi, M. Kumar, and V. K. Shahi, Membrane-based techniques for the separation and purification of proteins: An overview, Adv. Colloid Interface Sci., 145 (2009), pp. 1-22.

[45] D. D. SchmidT, Kinetic Monte Carlo Methods for Computing First Capture Time Distributions in Models of Diffusive Absorption, Harvey Mudd College Senior Thesis, Claremont, CA, 2017.

[46] D. Shoup And A. Szabo, Role of diffusion in ligand binding to macromolecules and cell-bound receptors, Biophys. J., 40 (1982), pp. 33-39.

[47] B. Smitha, S. Sridhar, and A. Khan, Solid polymer electrolyte membranes for fuel cell applications - a review, J. Membr. Sci., 259 (2005), pp. 10-26.

[48] I. N. Sneddon, Elements of Partial Differential Equations, McGraw-Hill, 1957.

[49] E. M. Stein and R. Shakarchi, Complex Analysis, Princeton Lect. Anal. 2, Princeton University Press, 2003.

[50] W. Strieder, Interaction between two nearby diffusion-controlled reactive sites in a plane, J. Chem. Phys., 129 (2008), 134508.

[51] A. Y. TOUkmaJi And J. A. BoARd, Ewald summation techniques in perspective: A survey, Comput. Phys. Commun., 95 (1996), pp. 73-92.

[52] R. Varma, G. Campi, T. Yokosuka, T. Saito, and M. L. Dustin, T cell receptor-proximal signals are sustained in peripheral microclusters and terminated in the central supramolecular activation cluster, Immunity, 25 (2006), pp. 117-127.

[53] M. J. Ward, W. D. Henshaw, and J. B. Keller, Summing logarithmic expansions for singularly perturbed eigenvalue problems, SIAM J. Appl. Math., 53 (1993), pp. 799-828, https://doi.org/10.1137/0153039.

[54] M. J. Ward And J. B. Keller, Strong localized perturbations of eigenvalue problems, SIAM J. Appl. Math., 53 (1993), pp. 770-798, https://doi.org/10.1137/0153038.

[55] J.-C. WU AND S.-Y. Lu, Patch-distribution effect on diffusion-limited process in dilute suspension of partially active spheres, J. Chem. Phys., 124 (2006), 024911.

[56] R. Zwanzig, Diffusion controlled ligand binding to spheres partially covered by receptors: An effective medium treatment, Proc. Nat. Acad. Sci. U.S.A., 87 (1990), pp. 5856-5857. 